\title{
Interaction of CCR4-NOT with EBF1 regulates gene-specific transcription and mRNA stability in B lymphopoiesis
}

\author{
Cheng-Yuan Yang, ${ }^{1}$ Senthilkumar Ramamoorthy, ${ }^{1,3}$ Sören Boller, ${ }^{1,3}$ Marc Rosenbaum, ${ }^{1,3}$ \\ Alfonso Rodriguez Gil, ${ }^{1}$ Gerhard Mittler, ${ }^{1}$ Yumiko Imai, ${ }^{2}$ Keiji Kuba, ${ }^{2}$ and Rudolf Grosschedl ${ }^{1}$ \\ ${ }^{1}$ Department of Cellular and Molecular Immunology, Max Planck Institute of Immunobiology and Epigenetics, \\ 79108 Freiburg, Germany; ${ }^{2}$ Department of Biochemistry and Metabolic Sciences, Akita University Graduate School of Medicine, \\ Akita 010-8543, Japan
}

Transcription factor EBF1 (early B-cell factor 1) regulates early B-cell differentiation by poising or activating lineagespecific genes and repressing genes associated with alternative cell fates. To identify proteins that regulate the diverse functions of EBF1, we used SILAC (stable isotope labeling by amino acids in cell culture)-based mass spectrometry of proteins associated with endogenous EBF1 in pro-B cells. This analysis identified most components of the multifunctional CCR4-NOT complex, which regulates transcription and mRNA degradation. CNOT3 interacts with EBF1, and we identified histidine 240 in EBF1 as a critical residue for this interaction. Complementation of $E b f 1^{-/-}$progenitors with EBF1H240A revealed a partial block of pro-B-cell differentiation and altered expression of specific EBF1 target genes that show either reduced transcription or increased mRNA stability. Most deregulated EBF1 target genes show normal occupancy by EBF1H240A, but we also detected genes with altered occupancy, suggesting that the CCR4-NOT complex affects multiple activities of EBF1. Mice with conditional Cnot3 inactivation recapitulate the block of early B-cell differentiation, which we found to be associated with an impaired autoregulation of $E \boldsymbol{E} f \mathbf{f}$ and reduced expression of pre-B-cell receptor components. Thus, the interaction of the CCR4-NOT complex with EBF1 diversifies the function of EBF1 in a context-dependent manner and may coordinate transcriptional and post-transcriptional gene regulation.

[Keywords: CCR4-NOT complex; CNOT3; EBF1; transcription; mRNA stability; B-cell differentiation]

Supplemental material is available for this article.

Received June 13, 2016; revised version accepted October 4, 2016.

B lymphopoiesis converts multipotent hematopoietic progenitors into highly specialized antibody-secreting effector cells. Multipotent progenitors (MPPs), which represent a branching point between myeloid and lymphoid lineages, differentiate via lymphoid-primed MPPs (LMPPs) into common lymphoid progenitors (CLPs). This process of differentiation is accompanied by a progressive restriction of lineage potential by which CLPs can still generate all adaptive and innate lymphoid cells but no myeloid or erythroid cells (Kondo et al. 1997; Adolfsson et al. 2005). In CLPs (consisting of all lymphoid progenitors [ALPs] and B-cell-biased lymphoid progenitors [BLPs]) as well as pre-pro-B cells, multilineage priming of enhancers has been implicated in setting a chromatin state that facilitates the activation of the B-lineage program (Inlay et al. 2009; Mercer et al. 2011; Zandi et al.

\footnotetext{
${ }^{3}$ These authors contributed equally to this work Corresponding author: grosschedl@ie-freiburg.mpg.de Article published online ahead of print. Article and publication date are online at http://www.genesdev.org/cgi/doi/10.1101/gad.285452.116. Freely available online through the Genes \& Development Open Access option.
}

2012). B-cell programming requires a complex network of transcription factors (TFs) in which feed-forward loops and synergistic and antagonistic actions allow for a robust implementation of the B-cell program (Zandi et al. 2008; Lin et al. 2010; Mansson et al. 2012; Boller and Grosschedl 2014; Singh et al. 2014). In pro-B cells, the repression of genes associated with alternative cell fates stabilizes the lineage decision and commits the cells to the B-cell fate (for review, see Nutt and Kee 2007; Ramírez et al. 2010; Boller and Grosschedl 2014). Rearrangements of the immunoglobulin $\mu$ (Ig $\mu)$ heavy chain locus generate pre-B cells that express the pre-B-cell receptor (pre-BCR), consisting of the $\mu$ chain and the $\lambda 5$ (Igll1) and VpreB surrogate light chains (for review, see Chowdhury and Sen 2004). Further rearrangements of the Ig light chain loci generate immature B cells that express the BCR and

(C) 2016 Yang et al. This article, published in Genes \& Development, is available under a Creative Commons License (Attribution-NonCommercial 4.0 International), as described at http://creativecommons.org/licenses/by-nc/4.0/. 
leave the bone marrow for further differentiation in the spleen.

Early B-cell factor 1 (EBF1) plays a critical role in establishing the B-lineage program. Targeted gene inactivation results in a complete block at the onset of B-cell differentiation (Lin and Grosschedl 1995). Moreover, the forced expression of EBF1 in progenitors biases their lineage potential to the B-cell fate, and expression of EBF1 allows Ikaros-deficient progenitors to overcome their early block in differentiation and initiate the B-cell program (Pongubala et al. 2008; Reynaud et al. 2008; Banerjee et al. 2013). In addition, EBF1 collaborates with Pax5 in enforcing the B-lineage identity by repressing genes involved in alternative lineage programs (Nutt et al. 1999; Pongubala et al. 2008; Thal et al. 2009; Lukin et al. 2011; Banerjee et al. 2013; Nechanitzky et al. 2013).

EBF1 consists of an extended DNA-binding domain (DBD) with a structural homology with NF- $\mathrm{BB}$, an IPT (Ig-like, plexins, and TFs) domain, an HLH (helix-loop-helix) dimerization domain, and an unstructured C-terminal domain (Siponen et al. 2010; Treiber et al. 2010a). EBF1 regulates genes by activation and repression and by modulating the chromatin structure (for review, see Hagman et al. 2012; Boller and Grosschedl 2014). In particular, the binding of EBF1 to chromatin has been shown to correlate with dimethylation of Lys4 of histone $\mathrm{H} 3$ (Treiber et al. 2010b). In addition, the function of EBF1 has been associated with SWI/SNF-dependent remodeling of chromatin and DNA demethylation of some promoters (Maier et al. 2004; Gao et al. 2009; Boller et al. 2016). However, no physical interaction between EBF1 and SWI/SNF proteins has yet been demonstrated. Only a small number of interaction partners have been identified that regulate the activity of EBF1. In particular, the related zinc finger proteins Zfp423 (Oaz and Ebfaz) and Zfp521 (Evi3) interact with EBF1 and antagonize its transcriptional activation potential (Hata et al. 2000; Hentges et al. 2004; Kiviranta et al. 2013). In contrast to well-studied interactions of TFs with multiprotein complexes that affect the transcriptional activity or the chromatin structure (for review, see Schneider and Grosschedl 2007; Malik and Roeder 2010; Meier and Brehm 2014), much less is known about proteins that coordinate the regulation of gene expression between the nucleus and cytoplasm.

The CCR4-NOT complex is a 1.2- to 1.9-MDa multisubunit complex that is found in both the nucleus and cytoplasm and has been shown to play a role in gene transcription, mRNA degradation, and quality control during protein synthesis (for review, see Miller and Reese 2012; Collart et al. 2013). CCR4/NOT was initially identified as a transcriptional regulator (Collart and Struhl 1994; Liu et al. 1998; Zwartjes et al. 2004) and subsequently as a cytoplasmic deadenylase that shortens the poly(A) tail of mRNA (Tucker et al. 2001; Chen et al. 2002). Recently, the CCR4-NOT complex has also been shown to regulate transcription elongation (Kruk et al. 2011; Dutta et al. 2015) and cotranslational quality control by its associated ubiquitin E3 ligase activity (for review, see Panasenko 2014). The CCR4-NOT complex has been implicated in the promoter-mediated coordina- tion of transcription and mRNA decay by interacting with the Rpb4/7 subunits of RNA polymerase II (Lotan et al. 2005; Villanyi et al. 2014). The CCR4-NOT complex is assembled around the CNOT1 subunit, which serves as a scaffold and interacts with various modules. The CNOT2 and CNOT3 subunits together form a module engaged in gene transcription and RNA targeting, whereas CNOT6, CNOT6L, CNOT7, and CNOT8 form a "deadenylase module" involved in mRNA degradation (for review, see Miller and Reese 2012; Collart et al. 2013). In addition, CNOT4 represents a module for protein quality control that is not a constitutive component of the complex (Lau et al. 2009; Bhaskar et al. 2015). Thus, multiple modules with distinct functional activities are tethered to CNOT1, forming a multisubunit complex that helps to coordinate different events in gene expression (for review, see Collart et al. 2013). However, insights into the targeting of the CCR4-NOT complex to specific sites in the genome have been very limited.

\section{Results}

\section{EBF1 interacts with the CCR4-NOT complex}

To identify novel interaction partners of EBF1, we performed a SILAC (stable isotope labeling by amino acids in cell culture)-based mass spectrometric analysis of proteins that are coimmunoprecipitated with endogenous EBF1 in pro-B cells. To this end, we immunoprecipitated EBF1 from 38B9 pro-B cells labeled with either heavy or light amino acids and compared the proteome of the eluate with that of an eluate from an immunoprecipitation with a control antibody. To minimize false positives, we performed six independent experiments in which we used forward and reverse labeling modes, different antiEBF1 antibodies, and benzonase treatment of the lysates to remove nucleic acids and thus exclude DNA- and RNA-mediated interactions. The overlap of proteins that were consistently enriched in all six experiments was visualized in a scatter chart and included a set of proteins in which most subunits of the CCR4-NOT complex are represented (Fig. 1A,B).

To confirm the results of the mass spectrometry of EBF1-interacting proteins and determine the key subunit of the CCR4-NOT complex interacting with EBF1, we expressed Strep-Flag-tagged EBF1 (EBF1-SF) together with individual HA-tagged CNOT subunits (CNOT2, CNOT3, CNOT6, CNOT7, and CNOT10) in HEK293 cells. Immunoblot analysis of cell lysates of the transfected cells revealed that all five CNOT subunits were expressed at similar levels (Fig. 1C). Strep tag pull-downs of EBF1 and EBF1-associated proteins from the cell lysates and subsequent immunoblot analysis to detect the HAtagged CNOT subunits indicated that EBF1 interacts with CNOT3 (Fig. 1C). To examine this interaction at physiological protein levels, we immunoprecipitated endogenous EBF1 from 38B9 pro-B-cell lysates and found coimmunoprecipitation of endogenous CNOT3 (Fig. 1D). Taken together, these results indicate that EBF1 interacts with the CNOT3 subunit of the CCR4-NOT 


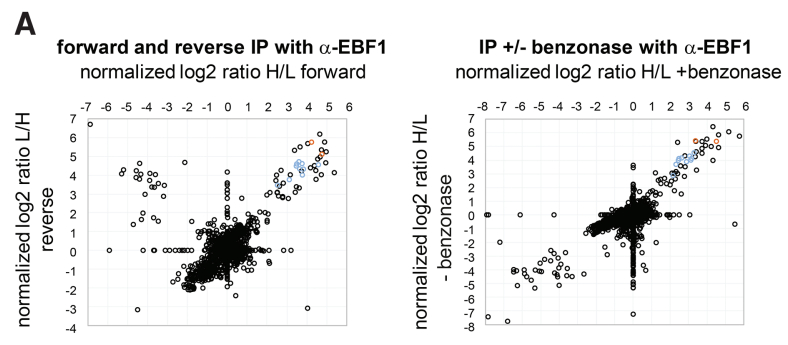

B

\begin{tabular}{lcccc}
\hline Protein & Ratio H/L & Ratio L/H & $\begin{array}{c}\text { Ratio H/L } \\
\text { - benzonase }\end{array}$ & $\begin{array}{c}\text { Ratio } \mathrm{H} / \mathrm{L} \\
\text { +benzonase }\end{array}$ \\
\hline EBF1 & 26.9 & 35.2 & 41.4 & 22.5 \\
EBF1 & 18.9 & 54.1 & 42.6 & 10.3 \\
\hline CNOT2 & 13.5 & 24.6 & 23.3 & 9.8 \\
\hline CNOT6I & 12.5 & 20.3 & 17.5 & 9.0 \\
\hline CNOT1 & 14.2 & 20.1 & 16.0 & 8.7 \\
\hline CNOT3 & 23.9 & 23.6 & 19.6 & 8.3 \\
\hline CNOT6 & 5.7 & 11.1 & 14.7 & 7.4 \\
\hline CNOT9 & 12.1 & 26.4 & 17.8 & 6.1 \\
\hline CNOT8 & 11.2 & 24.4 & 17.3 & 5.6 \\
\hline CNOT7 & 11.1 & 22.6 & 15.8 & 5.4 \\
\hline CNOT10 & 13.7 & 16.2 & 12.9 & 5.0 \\
\hline CNOT11 & 8.7 & 13.6 & 7.3 & 4.5
\end{tabular}

C

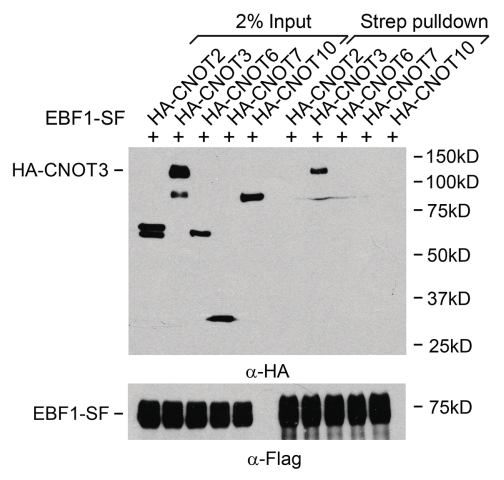

D

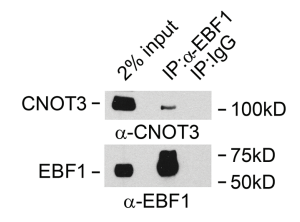

Figure 1. Identification of EBF1-interacting proteins. $(A)$ Scatter plots of SILAC-supported mass spectrometric analysis of proteins that are coimmunoprecipitated from $38 \mathrm{~B} 9$ pro-B-cell lysates with anti-EBF1 (1C) antibody. Scatter plots show data of forward versus reverse SILAC labeling (left) and data in the absence versus presence of benzonase (right). $\log _{2}$ values of normalized SILAC ratios of sample/control are shown. EBF1 polypeptides are depicted in orange, and members of the CCR4-NOT complex are shown in light blue. All other identified proteins are shown in black. $(B)$ SILAC ratios of enrichment of EBF1 and members of the CCR4-NOT complex in four independent experiments shown in $A$. Numbers represent the normalized sample/control ratios. EBF1 and members of the CCR4-NOT complex are labeled in orange and light blue, respectively. (C) Immunoprecipitation and immunoblot analysis to detect an interaction between StrepFlag-tagged EBF1 (EBF1-SF) and individual HA-tagged subunits of the CCR4-NOT complex by Strep-Tactin pull-down from lysates of transfected HEK293 cells. (D) Coimmunoprecipitation of endogenous EBF1 and CNOT3 interaction in 38B9 pro-B cells. EBF1 and EBF1-associated proteins were immunoprecipitated with anti-EBF1 antibody, and the immunoblots were probed with anti-CNOT3 or anti-EBF1 antibodies. Anti-IgG was used as a control. complex and that the other subunits are associated with EBF1 via complex formation with CNOT3. Moreover, the detection of the EBF1:CCR4-NOT complex association in benzonase-treated lysates suggests that the interaction is not mediated via DNA or RNA.

\section{EBF1:CNOT3 interaction requires a specific residue in the $D B D$}

To examine the specificity of EBF1 interaction with CNOT3 and identify specific amino acids in EBF1 that mediate protein:protein contacts, we performed Strep tag pull-downs with lysates of HEK293 cells in which HA-CNOT3 was expressed together with wild-type or mutated forms of EBF1-SF. In particular, we deleted the $\mathrm{DBD}(\triangle \mathrm{DBD})$, short $\mathrm{N}$-terminal domain $(\Delta \mathrm{N})$, IPT domain $(\Delta \mathrm{IPT})$, or C-terminal domain $(\Delta \mathrm{C})$ (Fig. 2A). In the Strep tag pull-downs of EBF1 wild type (EBF1wt), $\Delta \mathrm{N}, \Delta \mathrm{IPT}$, and $\triangle \mathrm{C}$, we copurified HA-CNOT3 at similar levels after normalization to the expression of EBF1 (Fig. 2B). However, in the Strep tag pull-down of EBF1 $\triangle \mathrm{DBD}$, we detected HA-CNOT3 only at the background level observed with a lysate lacking EBF1-SF, although $\triangle \mathrm{DBD}$ was expressed more abundantly than $\triangle \mathrm{C}$ (Fig. 2B). Thus, CNOT3 interacts with the DBD of EBF1. Using further $\mathrm{N}$-terminal truncations of the EBF1 DBD, we delineated a region between amino acids 194 and 251 as being important for the interaction with CNOT3 (Fig. 2B). To rule out that the interaction of the EBF1 DBD with CNOT3 does not reflect a DNA-mediated association, we examined the mutation of R163A in EBF1, which abrogates the interaction of EBF1 with DNA (Treiber et al. 2010a; Hagman et al. 2012). No effect of the R163A mutation on the interaction of EBF1 with CNOT3 was observed (Fig. 2B), suggesting that the proteins associate via protein:protein contacts.

To identify specific amino acids that are involved in the EBF1:CNOT3 interaction, we used structure-guided mutations of the DBD of EBF1. Previous structural analysis of DNA-bound homodimeric EBF1 indicated that the DBD (amino acids 24-240) has a pseudo-Ig-like $\beta$-sandwich fold with a structural similarity to the Rel homology domain (Siponen et al. 2010; Treiber et al. 2010a). DNA binding by EBF1 involves three loops and a zinc knuckle, whereas other loops that connect $\beta$ sheets or connect the DBD with the IPT domain are potentially available for protein interactions (Treiber et al. 2010a). Based on the structure of DNA-bound EBF1, we introduced clustered alanine mutations into three loops: QSG (44-46), residing between an $\alpha$ helix and the first $\beta$ sheet; SMT(133-135), residing between the fifth $\beta$ sheet and the zinc knuckle; and GNRNE (171-175), residing between the zinc knuckle and the sixth $\beta$ sheet (Supplemental Fig. S1A). Moreover, we mutated the C-terminal SKH (238-240) motif of the DBD (Supplemental Fig. S1A). Coexpression of these mutants with CNOT3 in transfected HEK293 cells and subsequent Strep tag pull-downs indicated that the SKH-AAA mutation impaired the enrichment of CNOT3 as efficiently as the $\triangle \mathrm{DBD}$ mutation (Supplemental Fig. S1B). S238 and K239 form H bonds with DNA, whereas the aromatic imidazole ring of $\mathrm{H} 240$ is surface-exposed and may 
A

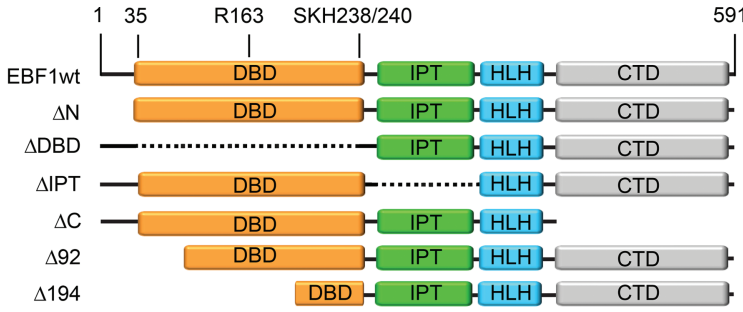

B
EBF1-SF
HA-CNOT3 HA-CNOT3

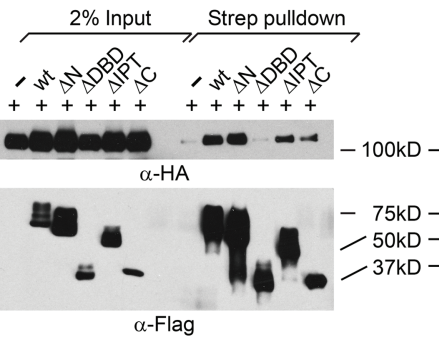

C
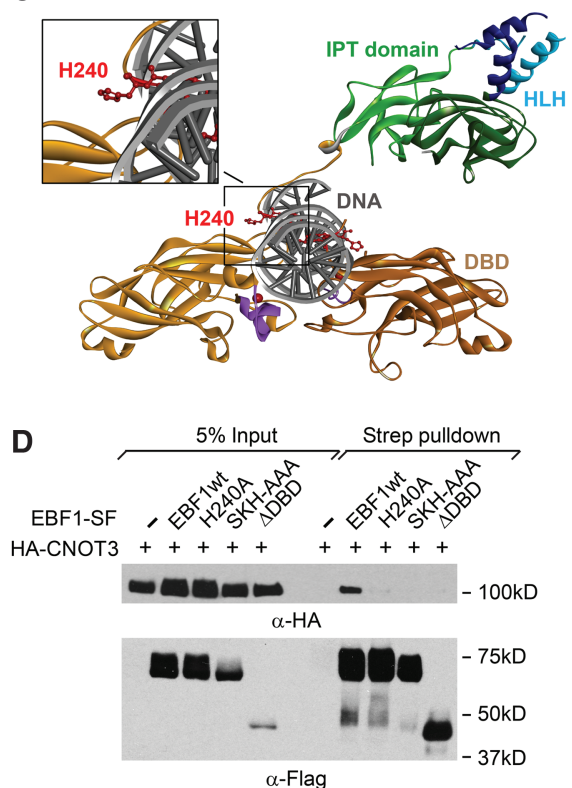

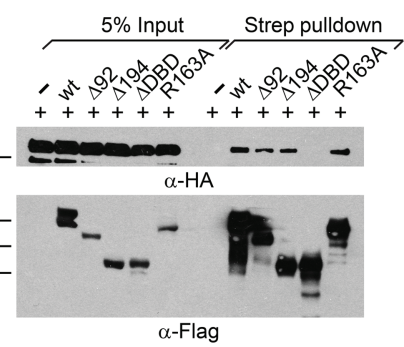

$E$
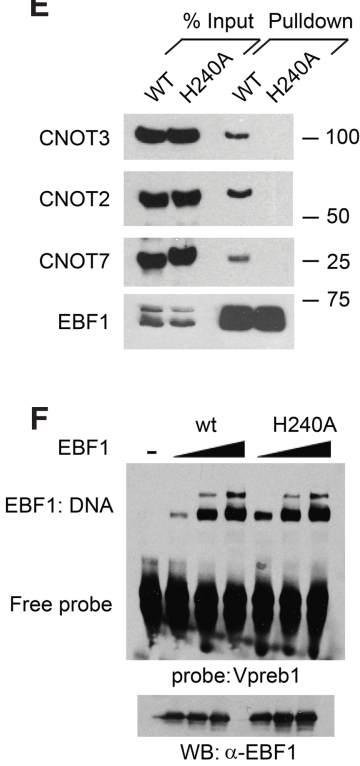

Figure 2. Delineation of amino acids in EBF1 that are involved in the interaction with CNOT3. (A) Schematic representation of EBF1 deletions and point mutations used to delineate the EBF1:CNOT interaction. The DBD and IPT domains are represented in orange and green, respectively. HLH and CTD denote the HLH domain (blue) and the C-terminal domain (gray). Positions of amino acids that were mutated are shown above the scheme of EBF1wt. $(B-D)$ Delineation of EBF1 domains involved in the interaction with CNOT3. $(B)$ Coimmunoprecipitations of SF-tagged wild-type or mutated EBF1 proteins with $\mathrm{HA}$-tagged CNOT3 to detect interaction in transfected HEK293 cells. EBF1 and EBF1interacting proteins were pulled down from cell lysates using Strep-Tactin beads. EBF1 and CNOT3 were visualized by immunoblot analysis using anti-Flag and anti-HA antibodies, respectively. (C) Structure of the DNA-bound EBF1 (Treiber et al. 2010a), with the region of the surfaceexposed histidine at position 240 enlarged. $(D)$ Identification of amino acids in EBF1 involved in the interaction with CNOT3. Coimmunoprecipitations of EBF1-SF proteins carrying point mutations with HA-tagged CNOT3 to detect interaction in transfected HEK293 cells. The analysis of the interaction was performed as described in B. (E) Coimmunoprecipitations of EBF1wt and EBF1H240A to detect interaction with CCR4-NOT subunits in A-MuLV transformed pro-B cells in which the endogenous Ebf1 allele had been deleted. $(F)$ Analysis of the DNA-binding ability of EBF1wt and EBF1H240A proteins using an electrophoretic mobility shift assay with recombinant proteins and an oligonucleotide encompassing an EBF1-binding site in the VpreB1 gene. (Bottom panel) The use of similar amounts of EBF1wt and $\mathrm{EBF} 1 \mathrm{H} 240 \mathrm{~A}$ proteins was confirmed by immunoblot analysis. allow for protein interaction (Fig. 2C; Treiber et al. 2010a). Therefore, we generated the H240A mutation and found that this mutation is sufficient to abrogate the EBF1: CNOT3 interaction (Fig. 2D). To determine whether the mutation impairs the interaction with the entire CCR4NOT complex, we performed coimmunoprecipitation experiments with lysates of cells in which the endogenous EBF1 had been replaced by wild-type or H240A mutant EBF1-SF. To this end, we transduced A-MuLV transformed pro-B cells from Ebf1 $1^{f l / f l} R E R T^{C r e}$ mice with EBF1wt- or H240A-expressing retroviruses and deleted the endogenous Ebf1 gene by treatment of the cells with 4-hydroxy-tamoxifen (Boller et al. 2016). In EBF1H240Aexpressing cells, we observed a virtual loss of interaction with two additionally examined subunits of the CCR4NOT complex: CNOT2 and CNOT7 (Fig. 2E). We also examined whether the H240A mutation alters the DNAbinding ability of EBF1. Therefore, we performed an elec- trophoretic mobility shift assay with labeled oligonucleotides encompassing an EBF1-binding site in the VpreB1 gene and with recombinant EBF1wt or EBF1H240A. The similar DNA-binding efficiency of both proteins indicated that the histidine residue at 240 does not affect the DNA binding of EBF1 in vitro (Fig. 2F). Taken together, these data suggest that a surface-exposed histidine at the base of a flexible loop between the DBD and IPT domains is involved in the interaction of EBF1 with the CCR4-NOT complex via CNOT3.

The EBF1H240A mutation impairs cell differentiation and expression of target genes

The identification of a specific amino acid in EBF1 that mediates the interaction with the CCR4-NOT complex enabled us to investigate a putative EBF1-dependent role 
A
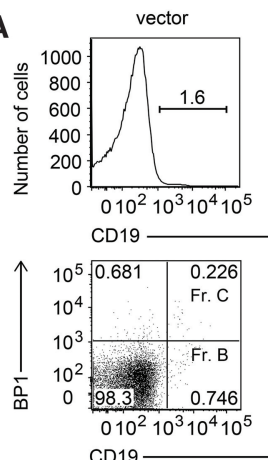

\section{B}

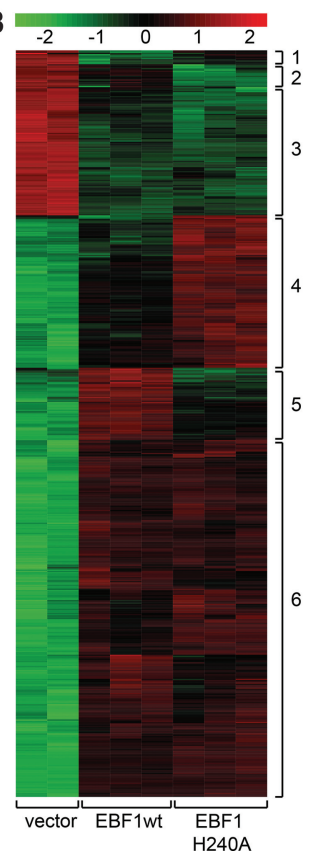

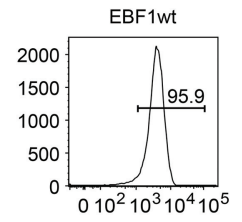

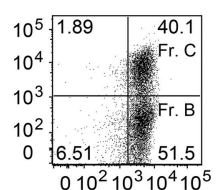

$010^{2} 10^{3} 10^{4} 10^{5}$

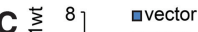

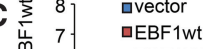

罚 6.

단.

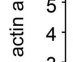

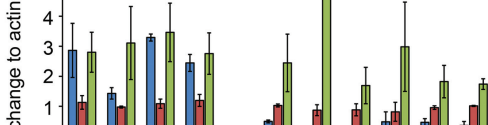

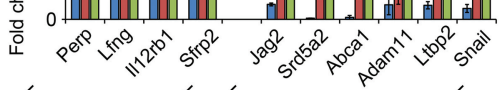

$$
\begin{gathered}
\text { Repressed by } \\
\text { EBF1wt (cluster } 1 \text { ) }
\end{gathered}
$$
EBF1wt (cluster 1)
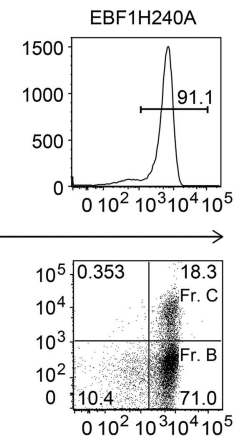

D

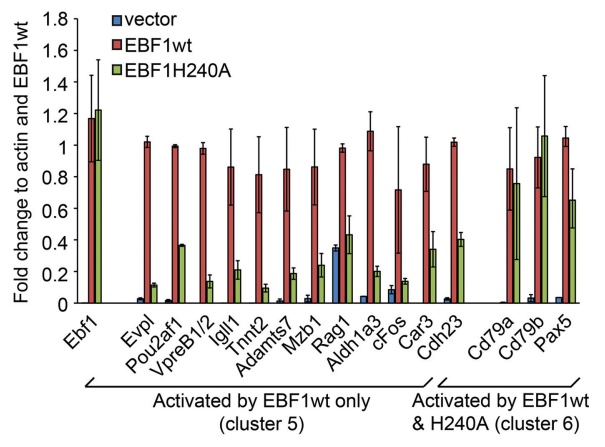

Figure 3. The H240A mutation impairs EBF1-mediated cell differentiation and gene expression. $(A)$ Flow cytometric analysis of B-cell differentiation in $E b f 1^{-/-}$progenitors transduced with bicistronic retroviruses expressing GFP alone or GFP together with EBF1wt or EBF1H240A. GFP-positive cells were gated for CD19 expression and analyzed for BP1 expression to distinguish early-pro-B (Fr. B) and late pro-B (Fr. C) cells. (B) Genome-wide microarray analysis to examine RNA expression in sorted Fr. B pro-B cells expressing EBF1wt or EBF1H240A. The heat map shows clusters of genes that are occupied by EBF1, as determined by ChIP-seq (chromatin immunoprecipitation [ChIP] combined with high-throughput sequencing) analysis (Treiber et al. $2010 \mathrm{~b}$ ), and affected by the expression of EBF1wt or EBF1H240A relative to the empty vector control by a factor of at least two. Expression values were scaled to the $z$ score (top), and two to three independent biological replicates were analyzed (bottom). $(C, D)$ Quantitative RTPCR (qRT-PCR) analysis of representative genes in clusters 1, 4, 5, and 6 in EBF1wt- and EBF1H240A-expressing Fr. B pro-B cells. The type of regulation of the genes (repressed by EBF1wt, enhanced by EBF1H240A, activated by EBF1wt only, and activated by EBF1wt and 240A) is indicated. Raw cycle values were normalized to actin and are represented as fold expression to one replicate of EBF1wt. Error bars represent standard deviation (SD) of three biological replicates. of this ubiquitously expressed and multifunctional protein complex in B-cell differentiation and gene expression. To this end, we transduced bicistronic retroviruses expressing EBF1wt or EBF1H240A along with GFP into $E b f 1^{-1-}$ progenitors that are arrested at the pre-pro-B-cell stage and examined the appearance of CD19-positive cells by flow cytometry. After $6 \mathrm{~d}$ of culture, almost all $\mathrm{GFP}^{+}$ cells of EBF1wt or EBF1H240A transduced cells expressed CD19, indicating that the function of EBF1 in the specification of the B-cell lineage is independent of its interaction with CNOT3 (Fig. 3A). As a control, virtually none of $\mathrm{GFP}^{+}$cells of vector transduced cells was positive for CD19. Further flow cytometric analysis showed that the ratio of $\mathrm{CD} 19^{+} \mathrm{BP}^{-}$early pro-B cells to $\mathrm{CD} 19^{+} \mathrm{BP} 1^{+}$late pro-B cells is increased in EBF1H240A-expressing cells relative to EBF1wt transduced cultures (Fig. 3A). We examined the proliferation and survival of EBF1wt- and EBF1H240A-expressing $\mathrm{CD} 19^{+} \mathrm{BP} 1^{+}$pro-B cells by measuring the incorporation of EdU and AnnexinV, respectively. Cell proliferation was found to be similar in EBF1wt- and EBF1H240A-expressing pro-B cells, and the frequency of apoptotic cells was modestly reduced in
EBF1H240A-expressing pro-B cells (Supplemental Fig. S1C,D). Thus, the H240A mutation of EBF1 results in an impaired differentiation of early to late pro-B cells.

To examine the effects of the H240A mutation on EBF1regulated gene expression prior to the partial developmental block in long-term cell cultures, we performed microarray-based transcriptome analysis on EBF1wt or EBF1H240A transduced Ebf1-/- progenitor cells $36 \mathrm{~h}$ after transduction. We compared the transcriptomes of triplicate cultures and considered only genes that are bound by EBF1 in the 38B9 pro-B-cell line, as determined by ChIP-seq (chromatin immunoprecipitation [ChIP] combined with high-throughput sequencing) analysis (Treiber et al. 2010b), and show more than a twofold difference in gene expression relative to vector transduced cells (Fig. 3B). A small cluster of seven genes (cluster 1), including Il12rb1 and Sfrp2, shows impaired down-regulation in EBF1H240A transduced cells relative to EBF1wt transduced cells (Fig. 3B,C). A larger cluster of 50 genes (cluster 5), including VpreB1, Igll1 (Lambda5), Rag1, Pou2af1 (OcaB), Mzb1, and cFos, represents genes that are less efficiently up-regulated by EBF1H240A relative to EBF1wt 
(Supplemental Table S1). Finally, two large clusters include genes that are similarly down-regulated (cluster 3; 80 genes, including Tcf7, Gfilb, and Ciita) or up-regulated (cluster 6; 227 genes total, including Cd79a, Cd79b, Pax5) in both EBF1wt- and EBF1H240A-expressing cells (Fig. 3B, D; Supplemental Table S1). We confirmed the changes in the expression of several genes from the clusters by quantitative RT-PCR (qRT-PCR) (Fig. 3C,D). Together, these data indicate that specific sets of EBF1-bound genes are deregulated in cells expressing EBF1H240A, in which the mutation affects both EBF1-activated and EBF1-repressed genes.

The H240A mutation alters the expression of EBF1 target genes by both transcriptional and post-transcriptional mechanisms

The dual roles of the CCR4-NOT complex in transcription and mRNA degradation prompted us to examine whether the altered expression of genes in EBF1H240Aexpressing pro-B cells can be accounted for by changes in mRNA stability and/or gene transcription. In particular, genes of the microarray cluster 4 (102 genes), which show enhanced expression in EBF1H240A transduced cells relative to EBF1wt transduced cells, could be regulated by CCR4-NOT-dependent mRNA degradation
(Fig. 3B,C). To this end, we incubated EBF1wt- and EBF1H240A-expressing cells with actinomycin D (Act D) to block transcription by RNA polymerase II and measured the mRNA levels of representative genes by qRT-PCR at $0,2,4$, and $8 \mathrm{~h}$ after Act D treatment. Consistent with the microarray and qRT-PCR results, the stability of mRNAs of the cluster 4 genes Jag2, Heyl, and Il6ra was modestly but reproducibly higher in EBF1H240A-expressing cells than in EBF1wt-expressing cells (Fig. 4A). In contrast, the $A c t 1$ control gene, $C d 79 a$, and $C d 79 b$, representing genes of cluster 6 (227 genes), and Evpl, VpreB1, and $I g 111$, representing cluster 5 (50 genes), showed no significant changes in mRNA stability (Fig. 4A). Thus, a relatively small subset of genes that are bound and regulated by EBF1 showed an increased mRNA half-life in EBF1H240A- versus EBF1wt-expressing cells.

The observed decreases in mRNA expression of cluster 5 genes in EBF1H240A versus EBF1wt transduced cells, which are not accompanied by significant changes in mRNA stability (Fig. 4A), raised the question of whether EBF1H240A has a defect in chromatin binding and/or transcriptional activation. We used quantitative ChIP to examine the occupancy of EBF1-binding sites associated with selected genes of cluster 5 and cluster 6 in EBF1H240A- and EBF1wt-expressing cells. Igll1, Mzb1, and Myl4 showed no significant differences in binding

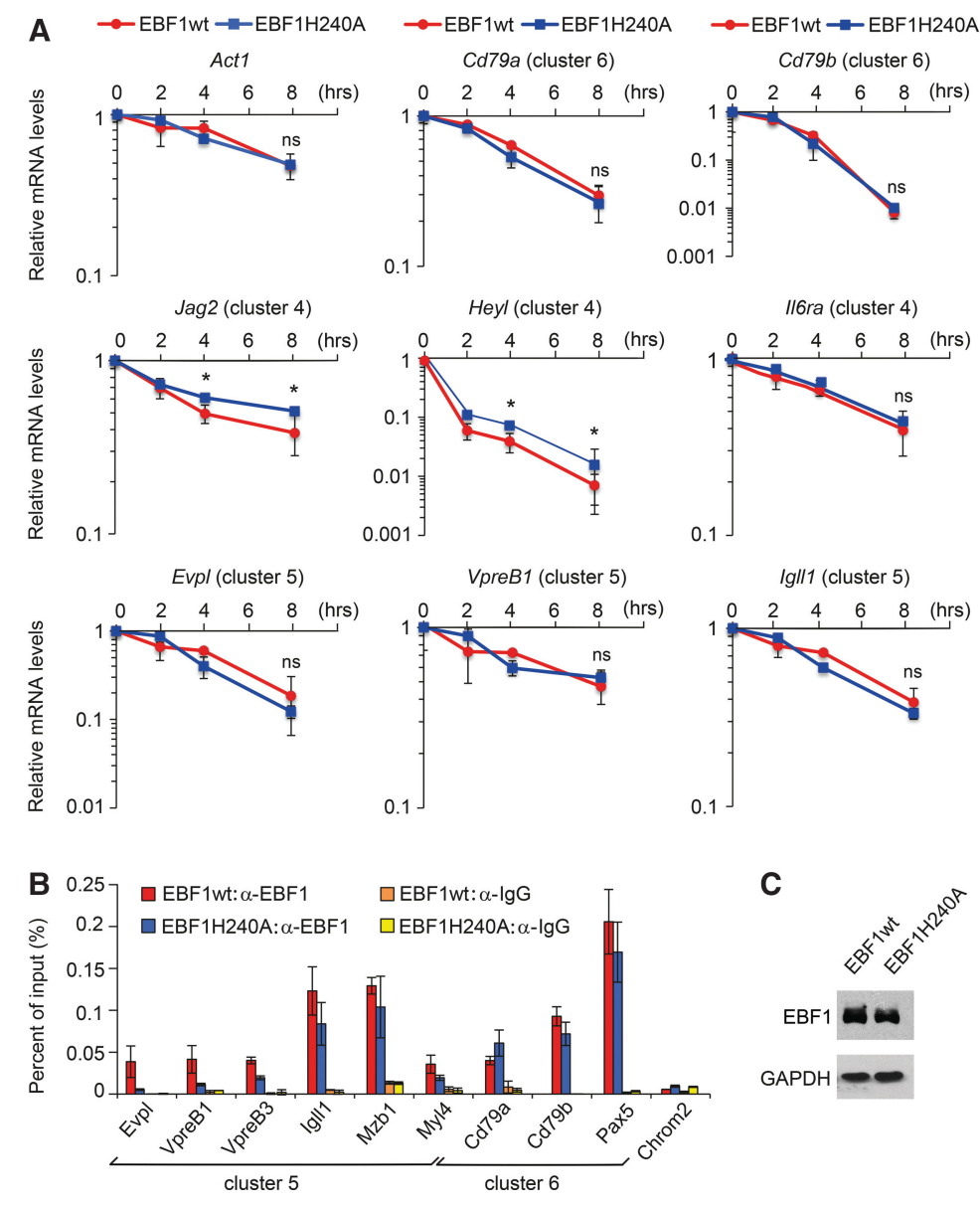

Figure 4. The H240A mutation results in gene-specific effects on mRNA stability and EBF1 occupancy. (A) Analysis of mRNA stability in $E b f 1^{-/}$progenitors transduced with EBF1wt- and EBF1H240A-expressing GFP bicistronic retroviruses. Cells sorted for the expression of GFP and CD19 were treated with Act D for $2 \mathrm{~h}$, and RNA was extracted at $0,2,4$, and $8 \mathrm{~h}$ after treatment. The mRNA amounts were determined by qRT-PCR. Raw cycle values were converted to absolute numbers using a gene standard curve and are presented as fold change relative to each group's time point 0 . Ebflwt and Ebf1H240A transcript levels are represented by red and blue lines, respectively. Error bars represent the SD of three independent experiments. $(B)$ ChIP analysis to examine EBF1 occupancy at EBF1 targets in EBF1wtand EBF1H240A-expressing sorted pro-B cells. An intergenic region of chromosome 2 and immunoprecipitation with IgG served as negative controls. Data are represented as percentage of input. Error bars represent the SD of three independent experiments. $(C)$ Immunoblot analysis to assess EBF1 expression in transduced and sorted pro-B cells. GAPDH was used as a loading control. 
EBF1wt and EBF1H240A proteins were found to have a similar half-life of 11 and $9 \mathrm{~h}$, respectively (data not shown).

To assess chromatin binding of EBF1wt and EBF1H240A in A-MuLV transformed pro-B cells on a global scale, we performed ChIP-seq analysis. This analysis indicated that 2192 EBF1 target sites were equally occupied by EBF1wt or EBF1H240A, whereas 2176 sites were occupied more efficiently by EBF1wt relative to EBF1H240A (Fig. 5A-C). We also detected a small group of sites that were preferentially bound by EBF1H240A. Analysis of the peak intensities indicated that equal occupancy by EBF1wt and EBF1H240A correlates with strongly bound sites (Fig. 5D, blue line), whereas weakly bound sites tend to be less efficiently occupied by EBF1H240A (Fig. $5 \mathrm{D}$, red line). The bioinformatic analysis of EBF1-binding sites and neighboring TF-binding sites revealed the presence of Ets/Pu.1 and Runxl motifs in all three groups (Fig. 5E; Supplemental Fig. S3A). In the wild-type >
G

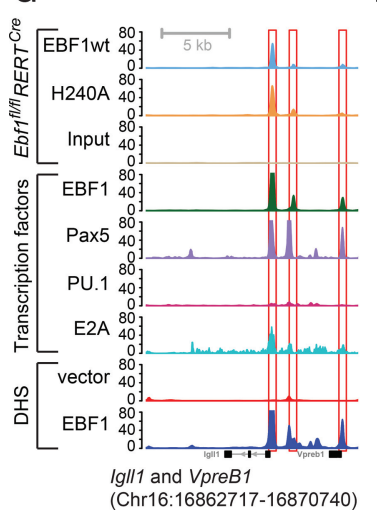

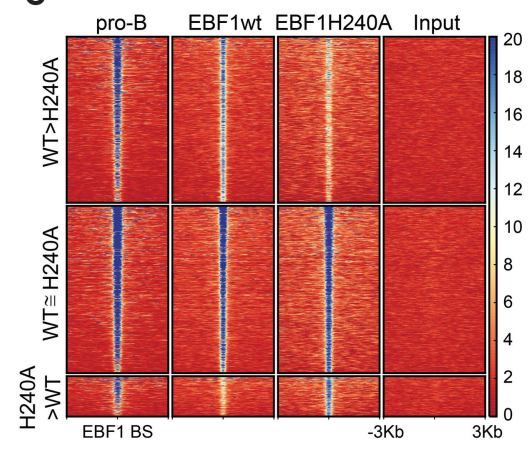

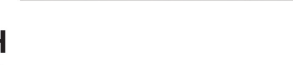

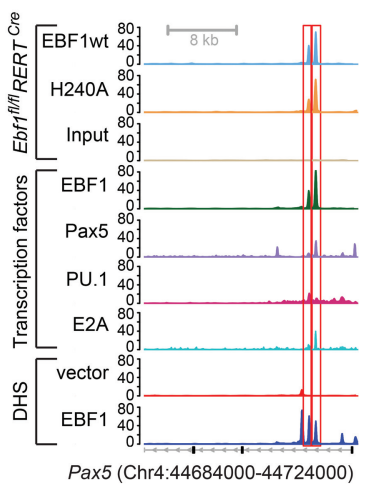

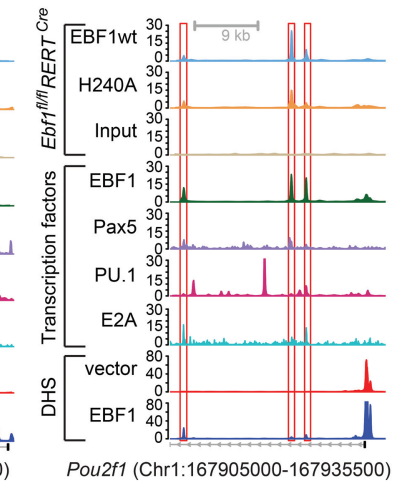

Figure 5. Genome-wide ChIP-seq analysis of EBF1wt and EBF1H240A. (A) Venn diagram represents the overlap of EBF1wtand EBF1H240A-occupied peaks, as determined by ChIP-seq, in pro-B cells in which the endogenous EBF1 was replaced with EBF1wt or EBF1H240A. (B) Scatter plot of EBF1wt only (red), EBF1H240A only (green), and common (black) peak densities around \pm 150 base pairs (bp) of peak summit in EBF1wt ( $X$-axis) and EBF1H240A ( $Y$-axis) ChIP data. $(C)$ Distribution of EBF1 ChIP signals $\pm 3 \mathrm{~kb}$ around EBF1 peak centers in Rag2 $^{-/}$pro-B cells and EBF1wt- or EBF1H240A-expressing pro-B cells. The peaks are grouped into three clusters as described in $A$. The heat map densities represent the normalized RPGC (reads per genomic content) scores. $(D)$ Read coverage around $\pm 3 \mathrm{~kb}$ of EBF1 peak centers in EFB1wt and EBF1H240A ChIP data sets. The wild-type $>\mathrm{H} 240 \mathrm{~A}$, wild-type $\cong$ H240A, and H240A > wild-type peaks are highlighted in red, blue, and green, respectively. (E) The top five de novo motifs identified within \pm 75 bp from EBF1 peak centers in wild-type $\cong \mathrm{H} 240 \mathrm{~A}$ and wild-type > H240A clusters are shown. The best-predicted TF-binding sites, percentage of peaks having the motif, $P$-value, and percentage of abundance in background sequences are indicated for each motif. $(F)$ Relative percentages of TF co-occupancy (E2A, Pax5, IRF4, PU.1, and Ikaros) in wild-type > $\mathrm{H} 240 \mathrm{~A}$ and wild-type $\cong \mathrm{H} 240 \mathrm{~A}$ clusters. $(G-I)$ EBF1 occupancy in EBF1wt- or EBF1H240A-expressing Ebf1 ${ }^{f l / f 1} R E R T^{C r e}$ pro-B cells $($ top), TF co-occupancy (EBF1, Pax5, PU.1, and E2A) in pro-B cells (middle), and DNase I-hypersensitive site (DHS) signals in vector or EBF1wt transduced Ebf1-/- progenitor cells are shown for the Igll1-VpreB1 loci $(G)$, Pax5 locus $(H)$, and Pou2f1 (Oct1) locus (I). The EBF1-binding sites are highlighted by red boxes. 
H240A group, we detected an additional CTCF motif associated with $\sim 11 \%$ of EBF1-occupied sites and a reduced frequency of the Ets motif (Fig. 5E). In this group, we also noted a reduced overlap with genes that are deregulated in H240A-expressing cells and observed a reduced occupancy by EBF1H20A at genes with apparently normal expression (Supplemental Fig. S3B,C). In the small H240A > wild-type group in which we observed preferential EBF1H240A occupancy, we noted a significantly reduced frequency of the consensus EBF1 motif (Supplemental Fig. S3A). Analysis of the overlap of EBF1-occupied sites with other TF-occupied sites, as determined by published ChIP-seq data sets for Ikaros, Pu.1, E2A, Pax5, and IRF4 (Lin et al. 2010; Revilla et al. 2012; Schwickert et al. 2014), indicated that sites equally occupied by EBF1wt and EBF1H240A are enriched for co-occupancy by other TFs (Fig. 5F). Gene-specific analysis of EBF1wt and EBF1H20A occupancy combined with published data of DNase I hypersensitivity and other TF occupancy (Boller et al. 2016) confirmed the normal or reduced EBF1 occupancy in EBF1H240A-expressing cells (Fig. 5G-I; Supplemental Fig. S3D-F). Taken together, these data demonstrate that the impaired interaction of EBF1H240A with the CCR4-NOT complex results in gene-specific and context-dependent effects on EBF1 occupancy, transcriptional activation, and mRNA stability.

\section{Conditional CNOT3 inactivation impairs pro-B-to-pre-B-cell differentiation}

Previous analysis of mice deficient for Cnot3 showed that this subunit of the CCR4-NOT complex is important for stress-induced cardiac function and lipid metabolism (Neely et al. 2010; Morita et al. 2011). Moreover, the partial block of B-cell differentiation in EBF1H240A transduced pre-pro-B cells suggested an important role of the EBF1:CNOT3 interaction in vivo. To further examine the function of CNOT3 in the B-cell lineage, we analyzed various B-cell populations in the bone marrow and spleens of mice carrying a tamoxifen-inducible Cre transgene and floxed alleles of Cnot 3 in which exons 2 and 3 could be deleted (K Kuba and Y Imai, unpubl.). Flow cytometric analysis of $C n o t 3^{f l / f 1} R E R T^{C r e}$ mutant mice in which the Cnot3 gene was efficiently deleted after tamoxifen treatment indicated that the frequencies of $\mathrm{B} 220^{\text {int }} \mathrm{CD} 43^{-}$and $\mathrm{B} 220^{\text {int }} \mathrm{CD} 19^{+}$bone marrow pre-B cells were reduced relative to $\mathrm{Cnot}^{+/+}{ }^{\mathrm{RERT}}{ }^{\mathrm{Cre}}$ mice (Fig. 6A; Supplemental Fig. S4A). The frequency of B220 int ${ }^{2} D 43^{+}$ pro-B cells was modestly increased in $\operatorname{Cnot} 3^{f l / f l} R E R T^{\text {Cre }}$ mutant mice, consistent with a block in pro-B-to-pre$\mathrm{B}$-cell differentiation. Moreover, the altered ratio of $\mathrm{HSA}^{+} \mathrm{BP}^{-}$early pro-B cells and $\mathrm{HSA}^{+} \mathrm{BP}^{+}$late pro-B cells revealed an additional partial block in the generation of late pro-B cells (Fig. 6B). Determination of the total numbers of pro-B and pre-B cells in multiple mice confirmed the block of pro-B-to-pre-B-cell differentiation in Cnot $3^{f l / f 1} R E R T^{C r e}$ mutant mice, whereas the decrease in the numbers of pro-B-cells was not significant (Fig. 6C). The efficient deletion of the floxed Cnot3 exons was confirmed by PCR analysis of genomic DNA from $\mathrm{B} 220^{+} \mathrm{CD} 43^{+}$bone marrow cells (Fig. 6D). In the spleens of Cnot3 mutant mice, we observed normal frequencies of $\mathrm{CD} 19^{+} \mathrm{CD} 93^{-}$mature $\mathrm{B}$ cells and $\mathrm{CD} 21^{+} \mathrm{CD} 23^{-}$marginal zone $\mathrm{B}$ cells but a decrease in the number of $\mathrm{CD} 9^{+} \mathrm{CD} 3^{+}$transitional B cells (Fig. 6E; Supplemental Fig. S4B). Thus, CNOT3 is required for the efficient generation of pre-B and immature $\mathrm{B}$ cells.

Expression of an Mzb1 transgene alleviates the block of differentiation in $\mathrm{Cnot} 3^{\mathrm{fl} / \mathrm{fl}} \mathrm{mb} 1^{\mathrm{Cre}}$ mice

To examine whether the defects of B-cell differentiation are cell-intrinsic, we also analyzed mice in which the Cnot3 gene was specifically inactivated in the B-cell lineage by $m b 1^{C r e}$. Flow cytometric analysis of bone marrow from $C n o t 3^{f l / f l} \mathrm{mb1}^{\mathrm{Cre}}$ mice revealed a reduced frequency of early and late pro-B cells and a defect in the generation of $\mathrm{B} 220^{\text {int }} \mathrm{CD} 43^{-}$pre-B cells and immature B cells that is more severe than that observed in $\mathrm{Cnot}^{f l / f 1} R E R T^{\mathrm{Cre}}$ mice (Fig. 6F,H; Supplemental Fig. S4C). Moreover, the number of $\mathrm{B} 220^{\mathrm{hi}} \mathrm{CD} 43^{-}$recirculating B cells in the bone marrow and that of mature follicular B cells in the spleen were markedly reduced in $C n o t 3^{f l / f 1} \mathrm{mb1} 1^{\mathrm{Cre}}$ mice (Fig. 6F; Supplemental Fig. S4D,E).

The phenotypic differences of $C n o t 3^{f l / f 1} R E R T^{C r e}$ and $C n o t 3^{f l / f 1} m b 1^{C r e}$ mice reminded us of differences in the block of pro-B-to-pre-B-cell differentiation in mice carrying a germline-null mutation of the Mzb1 gene versus mice containing a floxed Mzb1 allele in combination with the $m b 1^{C r e}$ allele (Rosenbaum et al. 2014). A block of pro-B-to-pre-B-cell differentiation was observed only in the presence of the $m b 1^{C r e}$ allele, which induces genotoxic stress via abundant Cre accumulation in the nucleus because of optimized codon usage and the presence of a nuclear localization sequence (Hobeika et al. 2006; Rosenbaum et al. 2014). The Mzb1 gene is bound and differentially regulated by EBF1wt and EBF1H240A and is modestly but significantly down-regulated in Cnot3 knockout pro-B cells (Fig. 3D; data not shown). Therefore, the question arose of whether the block of pro-B-to-preB-cell differentiation could be alleviated by the forced expression of Mzb1. To this end, we crossed Cnot $3^{\text {fl/fl }}$ $m b 1^{C r e}$ mice with transgenic mice that express the $M z b 1$ transgene from an $I g$ promoter/enhancer cassette (Supplemental Fig. S4G). Indeed, the forced expression of $M z b 1$ in B-lineage cells of $\mathrm{Cnot}^{\mathrm{fl} / f 1} \mathrm{mb} 1^{\mathrm{Cre}} \mathrm{Mzb} 1^{\text {tg }}$ mice alleviated the developmental block of pro-B-to-preB-cell differentiation (Fig. 6G,I). Analysis of the total numbers of the early B-cell populations in multiple mice confirmed the significance of the rescue by the Mzb1 transgene (Fig. 6J,K). Moreover, the spleens of Cnot $3^{f l / f l}$ $m b 1^{C r e} M z b 1^{t g}$ mice had increased frequencies of $\mathrm{CD} 21^{+} \mathrm{CD} 23^{+}$follicular B cells and CD $21^{+} \mathrm{CD} 23^{-}$marginal zone B cells relative to $C n o t 3^{f l / f 1} \mathrm{mb1}{ }^{\mathrm{Cre}}$ mice (Supplemental Fig. S4E,F). Thus, the impaired Mzb1 expression in Cnot3-deficient pro-B cells together with the presence of the $m b 1^{C r e}$ allele may account at least in part for the enhanced defect of pro-B-to-pre-B-cell differentiation in $\mathrm{Cnot}^{\mathrm{fl} / \mathrm{fl}} \mathrm{mb1}{ }^{\mathrm{Cre}}$ mice. 

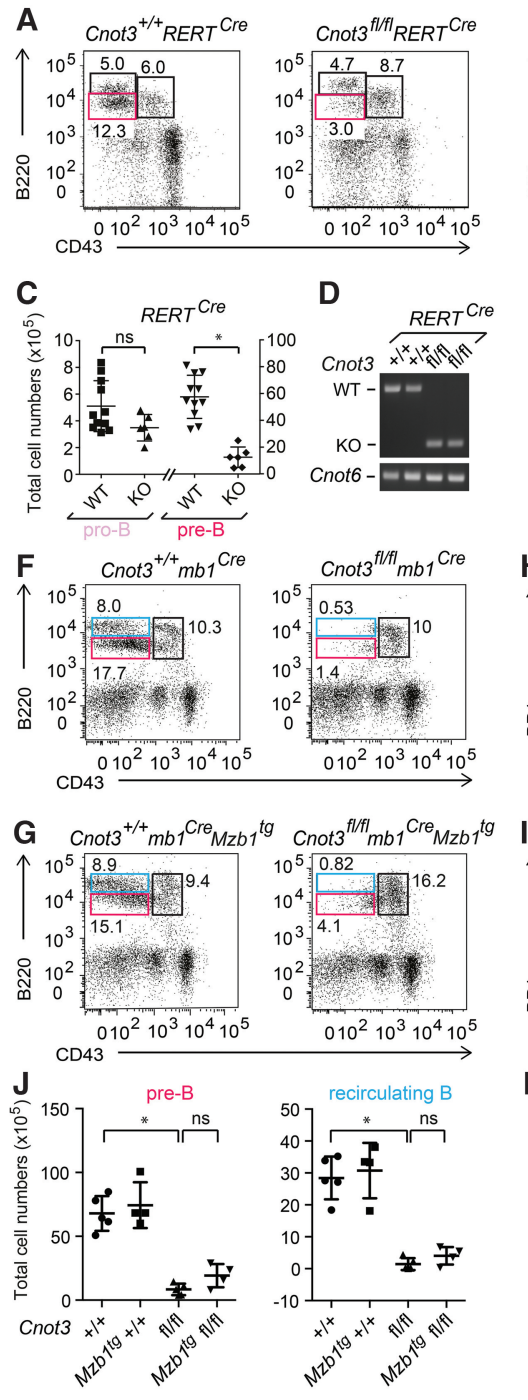
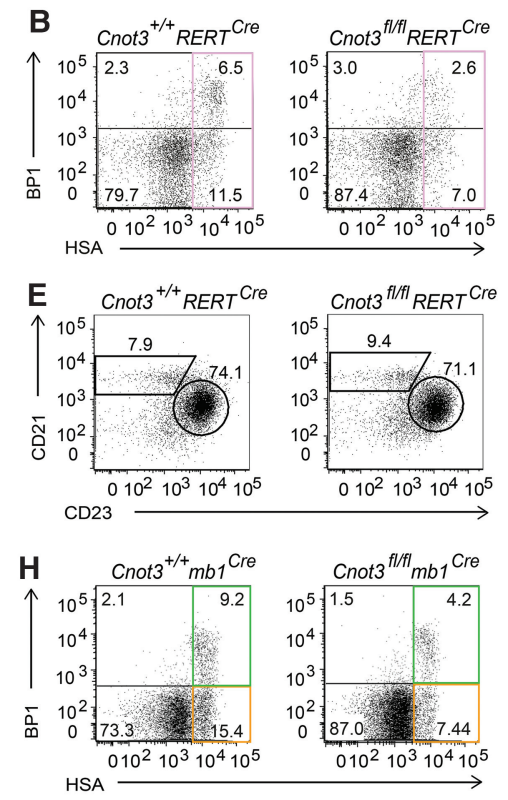

I $\mathrm{Cnot3}^{+/+}{ }_{\mathrm{mb}}{ }^{\mathrm{Cre}} \mathrm{Mzb}^{\mathrm{tg}} \mathrm{Cnot}^{\mathrm{flfff}}{ }_{\mathrm{mb} 1} \mathrm{Cre}_{\mathrm{Mzb}} 1^{\mathrm{tg}}$
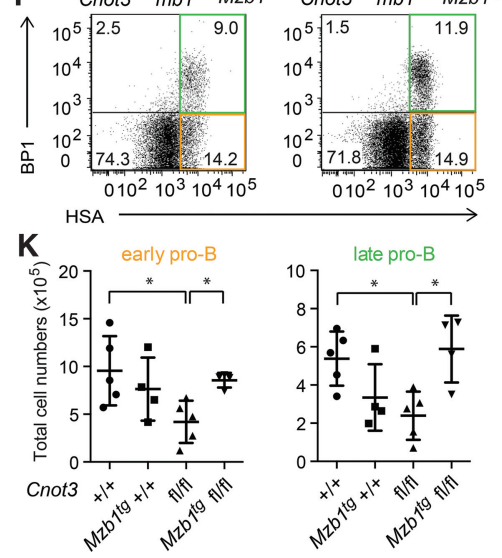

Figure 6. Cnot3-deficient mice show impaired early B-cell differentiation that can be alleviated in part by the expression of $M z b 1$. (A) Flow cytometric analysis of bone marrow $\mathrm{B}$ cells in $\mathrm{Cnot}^{+/+} R E R T^{\text {Cre }}$ and Cnot $3^{f 1 / f 1} R E R T^{\text {Cre }}$ mice to detect $\mathrm{B} 220^{+} \mathrm{CD} 43^{+}$pro-B, $\mathrm{B} 220^{\text {int }} \mathrm{CD} 43^{-}$late pre-B, and immature $\mathrm{B}$ cells as well as B220 ${ }^{\text {hi }} \mathrm{CD} 43^{-}$recirculating B cells in the living lymphocyte gate. $(B)$ Further gating of pro-B cells to detect pre-pro- $\mathrm{B}\left(\mathrm{HSA}^{-} \mathrm{BP}^{-}\right)$, early pro- $\mathrm{B}\left(\mathrm{HSA}^{+} \mathrm{BP}^{-}\right)$ and late pro- $\mathrm{B}\left(\mathrm{HSA}^{+} \mathrm{BP}^{+}{ }^{+}\right.$cells. (C) Statistical analysis of the total numbers of pro-B (pink) and pre-B (red) cells in $\mathrm{Cnot}^{+/+} \mathrm{RERT}^{\text {Cre }}$ (wild-type) and Cnot $3^{\text {fl/f1 }}$ $R E R T^{C r e}$ (knockout) mice. Long horizontal bars indicate the mean, and short horizontal bars represent the $\mathrm{SD}$ of the mean. Statistical significance between wild type and knockout is assessed by an unpaired two-tail Student's $t$-test. $(D)$ PCR analysis of genomic DNA from $\mathrm{Cnot}^{+/+} R E R T^{\mathrm{Cre}}$ and $\mathrm{Cnot} 3^{\text {fl/fl }}{ }^{\mathrm{R}} \mathrm{R} R T^{\mathrm{Cre}}$ mice to detect the deletion status of Cnot3. Cnot6 served as a loading control. (E) Flow cytometric analysis of splenic B cells to detect $\mathrm{CD} 21^{\text {int }} \mathrm{CD} 23^{+}$follicular B cells and $\mathrm{CD} 21^{\mathrm{hi}} \mathrm{CD} 23^{-}$marginal zone $\mathrm{B}$ cells in

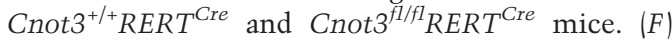
Flow cytometric analysis of bone marrow cells in $\mathrm{Cnot}^{+/+} \mathrm{mb1} 1^{\mathrm{Cre}}$ and $\mathrm{Cnot} 3^{\mathrm{fl} / \mathrm{fl}} \mathrm{mb1} 1^{\mathrm{Cre}}$ mice to detect the B-cell compartment as described in $A$. $(G)$ Flow cytometric analysis of the effects of a $M z b 1$ transgene expression $\left(M z b 1^{t g}\right)$ on early B-cell differentiation in $\mathrm{Cnot}^{+/+} \mathrm{mb1} 1^{\mathrm{Cre}}$ and $\mathrm{Cnot}^{\mathrm{fl} / \mathrm{fl}} \mathrm{mb1} 1^{\mathrm{Cre}}$ mice. $(\mathrm{H}, \mathrm{I})$ Gating of bone marrow $\mathrm{B} 220^{+} \mathrm{CD} 43^{+}$pro-B cells to detect $\mathrm{HSA}^{+} \mathrm{BP}^{-}$early pro-B and $\mathrm{HSA}^{+} \mathrm{BP}^{+}$late pro-B stages. $(J, K)$ Statistical analysis of the total numbers of pre-B (red), recirculating B (blue), early pro-B (orange), and late pro-B (green) cells in $\mathrm{Cnot}^{+/+}$ $\mathrm{mb1} 1^{\mathrm{Cre}}$, Cnot $3^{\mathrm{fl} / \mathrm{fl}} \mathrm{mb} 1^{\mathrm{Cre}}, \mathrm{Cnot}{ }^{+1_{+}} \mathrm{mb} 1^{\mathrm{Cre}} \mathrm{Mzb} 1^{\text {tg }}$, and $\mathrm{Cnot}^{f 1 / f 1} \mathrm{mb} 1^{\mathrm{Cre}} \mathrm{Mzb} 1^{\mathrm{tg}}$ mice. Numbers in the FACS profiles indicate the percentage of cells within the gated population. The data are representative of four or more independent experiments.

\section{CNOT3 deficiency impairs EBF1 autoregulation and pre-BCR expression}

To gain insight into the mechanism underlying the developmental defects in mice in which Cnot3 was conditionally deleted, we performed RNA sequencing (RNA-seq) analysis on two biological replicates of sorted $\mathrm{B} 22 \mathrm{O}^{+} \mathrm{CD} 43^{+}$pro-B cells from tamoxifen-treated Cnot3 $^{f l / f 1} R_{E R T}{ }^{\text {Cre }}$ (Cnot3 knockout) and $\mathrm{Cnot}^{+/+}$ $R E R T^{\text {Cre }}$ (Cnot3 wild-type) mice. The bioinformatic analysis confirmed the efficient loss of exons 2 and 3 in Cnot3 transcripts in Cnot3 knockout pro-B cells (Fig. 7A). In these mutant cells, 294 genes were up-regulated and 144 genes were down-regulated more than twofold relative to Cnot3 wild-type pro-B cells (Fig. 7B). Overlap of these RNA-seq data sets with EBF1 ChIP-seq data (Treiber et al. 2010b) indicated that $47 \%$ of down-regulated genes are occupied by EBF1, whereas $19 \%$ of up-regulated genes are bound by EBF1 (Fig. 7C). Notably, we observed impaired expression of Ebf1 and many EBF1 target genes, including Cd79a, Pax5, and Cd19 (Supplemental Table S2). To further examine the link between EBF1 and
CNOT3, we also overlapped this data set with the microarray data set of genes that are differentially regulated by EBF1wt- or EBF1H240A-expressing pro-B cells (Supplemental Table S1). Seven EBF1-bound genes, including

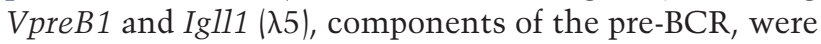
down-regulated in both Cnot3-deficient pro-B cells and EBF1H240A transduced progenitors relative to wildtype pro-B cells and EBF1wt transduced progenitors, respectively (Fig. 7D). However, many other EBF1-bound genes that were down-regulated in Cnot 3 knockout pro-B cells were expressed at similar levels in cells with forced expression of EBF1wt or EBF1H240A. Notably, the amounts of EBF1 and Pax 5 protein as well as the numbers of Ebf1 and Pax 5 transcripts were reduced in Cnot3-deficient pro-B cells (Fig. 7E; Supplemental Table S2). Pax 5 has been shown to regulate the use of distal V genes in $\mathrm{V}(\mathrm{D}) \mathrm{J}$ recombination (Fuxa et al. 2004). Consistent with the down-regulation of Pax5 in Cnot3deficient pro-B cells, we observed an impaired recombination of distal but not proximal $\mathrm{V}$ genes in the mutant cells (Fig. 7F). 
A

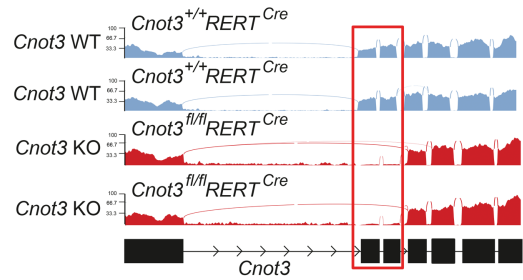

B

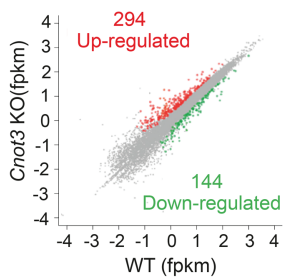

C

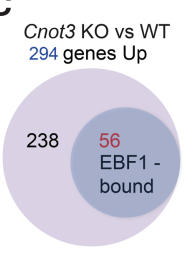

E
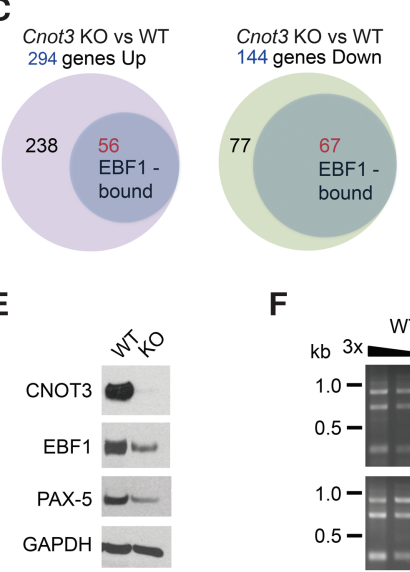

D

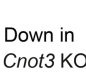
RNA-seq

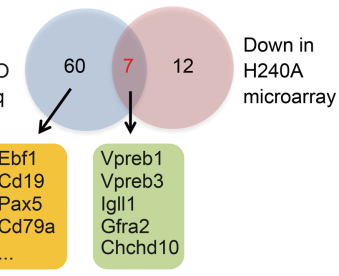

$\mathbf{F}$

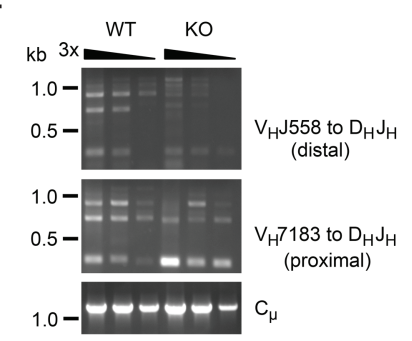

G
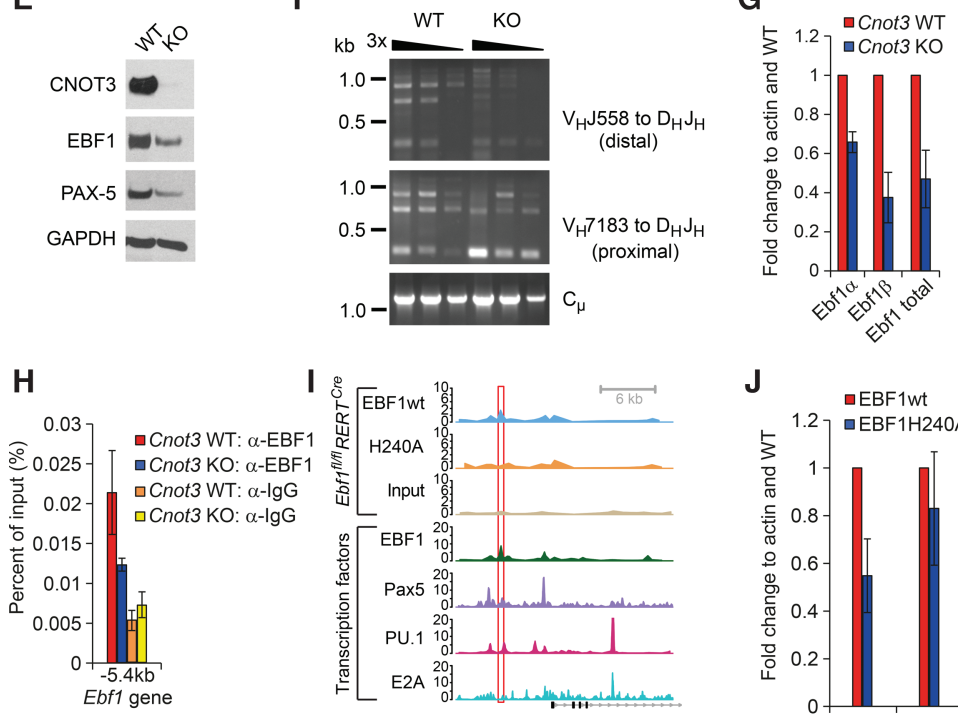

I

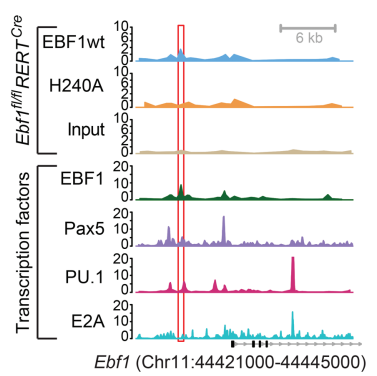

J

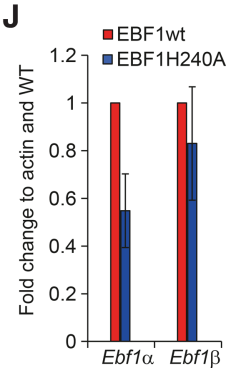

Figure 7. Inactivation of Cnot3 results in impaired autoregulation of Ebf1 and reduced expression of EBF1, Pax5, and components of the pre-BCR. (A) Sashimi blot depicting RNA-seq reads of Cnot3 transcripts in sorted pro-B cells from tamoxifen-treated $\mathrm{Cnot}^{+/+} R E R T^{\mathrm{Cre}}$ (wild-type) and $\mathrm{Cnot}^{\mathrm{fl} / \mathrm{fl}} \mathrm{RERT} \mathrm{T}^{\mathrm{Cre}}$ (knockout) mice. Exons 1-7 of the Cnot3 locus are shown. The absence of reads in exons 2 and 3 (highlighted by a red box) confirms the high efficiency of deletion in knockout pro-B cells. The scale is in FPKM (fragments per kilobase of exon per million mapped sequence reads). Data represent two biological replicates. $(B)$ Scatter plot of gene expression differences between Cnot3 wild-type and knockout pro-B cells. The up-regulated and down-regulated genes in knockout versus wild-type pro-B cells are indicated in red and green, respectively. $(C)$ Overlap of EBF1 occupancy, as determined by ChIP-seq analysis in pro-B cells (Treiber et al. 2010b), with up-regulation or down-regulation of genes upon Cnot3 deletion. Fifty-six of 294 up-regulated genes and 67 of 144 down-regulated genes are bound by EBF1. (D) Overlap of EBF1 occupany and down-regulation of genes in Cnot3 knockout pro-B cells, as determined by RNA-seq analysis, and EBF1H240A-expressing pro-B cells, as determined by microarray analysis. (E) Immunoblot analysis to detect CNOT3, EBF1, and Pax5 protein expression in pro-B cells from tamoxifen-treated $\mathrm{Cnot}^{+/+} R E R T^{\mathrm{Cre}}$ and $\operatorname{Cnot}^{\mathrm{fl} / \mathrm{fl}} R E R T^{\mathrm{Cre}}$ mice. GAPDH was used as a loading control. (F) Semiquantitative PCR analysis of genomic DNA from

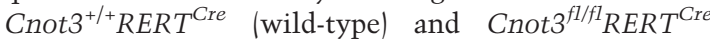
(knockout) pro-B cells to detect $\mathrm{V}(\mathrm{D}) \mathrm{J}$ rearrangements involving distal $\mathrm{V}_{\mathrm{H}} \mathrm{J} 558$ and proximal $\mathrm{V}_{\mathrm{H}} 7183$ genes. Threefold serial dilutions were used for PCR. C $\mu$ was used as a loading control. (G) qRT-PCR analysis to detect transcripts from the Ebf1a and Ebf1 $\beta$ promoters and all Ebf1 transcripts in Cnot3 wild-type and knockout pro-B cells. $(H)$ ChIP analysis to determine EBF1 occupancy at the Ebf1 locus in pro-B cells from Cnot3 wild-type or knockout mice. Results are represented as the percentage of input. IgG was used as an antibody control. Error bars represent the SD of three independent experiments. (I) Occupancy of the $E b f 1$ promoter region in $E b f 1^{f l / f 1} R E R T^{C r e}$ pro-B cells in which the endogenous EBF1 was replaced by EBF1wt or EBF1H240A. Occupancy by EBF1wt and EBF1H240A is shown at the top. Occupancy of the Ebf1 promoter region by EBF1, Pax5, PU.1, and E2A in pro-B cells is shown at the bottom. The EBF1 occupancy at -5.4 kb is highlighted by a red box. (J) qRT-PCR analysis of endogenous Ebf1a and Ebf1 $\beta$ transcripts in EBF1wt- or EBF1H240A-expressing pro-B cells.

In principle, the deregulation of many EBF1-bound genes in Cnot3-deficient pro-B cells could be accounted for by the reduced expression of EBF1, raising the question of a role of CNOT3 in the EBF1 autoregulatory feedback loop and/or EBF1-Pax5 positive feedback loop (Roessler et al. 2007; Decker et al. 2009). EBF1 binds to the distal Ebf1a promoter, whereas Pax5 binds to the proximal Ebf1 $\beta$ promoter (Smith et al. 2002; Roessler et al. 2007; Treiber et al. 2010b). Moreover, the forced expression of EBF1 in $E b f 1^{-/-}$progenitors enhances transcription from the endogenous Ebf1-null allele carrying a deletion of exon 3 and activates transcription of the Pax5 gene (Treiber et al. 2010b; Boller et al. 2016; data not shown). qRT-PCR of RNA from in vitro cultured tamoxifen-treated Cnot $3^{f l / f 1} R E R T^{\text {Cre }}$ pro-B cells confirmed the reduction of the number of Ebf1 transcripts (Fig. 7G). We also examined the transcript levels for both Ebf1a and Ebf1 $\beta$ isoforms and found that both isoforms were reduced in tamoxifen-treated $C$ not $3^{f l / f 1} R E R T^{\text {Cre }}$ pro-B cells relative to

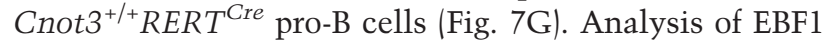
binding at cis-regulatory sequences of the genomic Ebf1 locus indicated that the occupancy at the EBF1-binding site present in the $-5.4-\mathrm{kb}$ distal promoter region is reduced in Cnot3-deficient pro-B cells relative to Cnot3 wild-type cells (Fig. 7H). Notably, in EBF1H240A-expressing pro-B cells, we observed a reduced EBF1 occupancy at the -5.4-kb distal Ebf1 a promoter site relative to the occupancy observed in EBF1wt-expressing cells (Fig. 7I). Moreover, the activation of the Ebf1a promoter by EBF1H240A was reduced relative to the activation by EBF1wt (Fig. 7J). Thus, efficient EBF1 binding at the -5.4-kb site of the distal Ebf1a promoter and autoregulation by EBF1 both appear to require the interaction of EBF1 with CNOT3. 


\section{Discussion}

Gene expression in eukaryotes requires transcription followed by splicing in the nucleus, export of mRNA to the cytoplasm for translation, and subsequent mRNA degradation. Recent studies in yeast and humans indicated that these processes are interconnected and that cis-acting sequences help to coordinate transcription and mRNA decay (Goler-Baron et al. 2008; Bregman et al. 2011). Moreover, these data implied that TFs regulate distinct processes of gene expression by interacting with multiple cofactors dedicated to individual steps in gene expression and/or with protein complexes that harbor multiple functional activities. Here we show that the cell type-specific TF EBF1 interacts with the multifunctional CCR4-NOT protein complex, which has been implicated in coordinating various steps in gene regulation.

The mammalian CCR4-NOT complex consists of multiple modules involved in transcription (CNOT2/ CNOT3), mRNA degradation (CNOT6/CNOT6L/ CNOT7/CNOT8), and protein quality control (CNOT4) (for review, see Miller and Reese 2012). We found that EBF1 interacts specifically with the CNOT3 subunit of the complex. However, most of the other subunits, with the exception of CNOT4, were identified in the mass spectrometric analysis of EBF1-associated proteins, suggesting that the CCR4-NOT complex lacking the protein quality control module interacts with EBF1.

The interaction of CNOT3 with EBF1, which involves a surface-exposed histidine in the EBF1 DBD, does not depend on the DNA-binding ability of EBF1. In particular, the R163A mutation of EBF1, which abrogates its ability to bind DNA, did not affect its interaction with CNOT3 in coimmunoprecipitation experiments. However, the interaction of EBF1 with the CCR4-NOT complex can enhance EBF1 occupancy at a specific set of target sites. Quantitative ChIP analysis and ChIP-seq analysis showed that the occupancy of $\sim 45 \%$ of sites is reduced in EBF1H240A-expressing cells relative to EBF1wt-expressing cells. A hallmark of these sites is a weak occupancy by EBF1 and lower co-occupancy by other TFs. Therefore, the interaction of the CCR4-NOT complex may indirectly enhance the occupancy of EBF1 at the "H240A-sensitive" sites. The sequences of "H240A-sensititive" and "H240A-insensitive" EBF1-binding sites are similar, suggesting that the chromatin context may influence the contribution of the CCR4-NOT complex to EBF1 occupancy in vivo. In line with our findings, DNA binding by RXRa was shown to be enhanced by its interaction with the CNOT7 subunit of the CCR4-NOT complex (Nakamura et al. 2004). CNOT7 interacts with a specific domain of RXRa in Sertoli cells, and DNA binding and transcriptional activation by $\mathrm{RXR} \alpha$ were found to be impaired in CNOT7-deficient testis extracts and CNOT7-deficient mouse embryonic fibroblasts, respectively (Nakamura et al. 2004). In embryonic stem cells, ChIP-seq analysis detecting CNOT3 occupancy revealed an enriched consensus sequence, 5'-CGGCNGCG, and suggested the possibility of sequence-specific binding by CNOT3 (Hu et al. 2009). However, the consensus site was found to overlap with the Trim 28 consensus binding site $5^{\prime}$ CGCGGC, and therefore it cannot be ruled out that CNOT3 had been cross-linked to specific genomic regions via an association with Trim28. Our bioinformatic analysis of EBF1-bound regions associated with genes that are affected by the EBF1H240A mutation did not identify any sequence that would resemble the 5'-CGGCNGCG motif. Thus, the interaction of the CCR4-NOT complex with TFs may help to target this multifunctional complex to specific genomic regions and stabilize the chromatin binding of TFs.

The H240A mutation affects the expression of specific sets of EBF1-bound and EBF1-regulated genes mostly without changing the EBF1 occupancy. Overlap of the ChIP-seq and microarray data sets indicated that the majority of genes that are deregulated in EBF1H240Aexpressing cells shows similar EBF1 occupancy in EBF1wt- and EBF1H240A-containing cells. In particular, a set of 177 EBF1-bound genes (microarray clusters 1, 2, 4 , and 5) is affected by the H240A mutation, whereas another set of 307 EBF1-bound genes (clusters 3 and $6)$ is unaffected. Notably, the effects of the H240A mutation on the expression of EBF1 target genes were diverse, including defects in gene repression (cluster 1), gene activation (cluster 5), and mRNA stability (cluster 4). Moreover, RNA-seq analysis in Cnot3-deficient pro-B cells indicated that $\sim 50 \%$ of down-regulated genes and $\sim 20 \%$ of up-regulated genes are occupied by EBF1. Thus, the regulation of a major proportion of genes by CNOT3 in pro-B cells appears to involve the function of EBF1.

The question arose as to how the recruitment of this multiprotein complex by EBF1 affects different steps in the expression of EBF1 target genes. Association of CCR4-NOT with promoters can result in either gene repression or activation (Winkler et al. 2006). Although the underlying mechanisms are still obscure, the effects on gene transcription may involve an interaction of CCR4-NOT with TFIID (Lemaire and Collart 2000; Sanders et al. 2002). The bioinformatic analysis of EBF1-bound regions linked with genes in which the interaction of EBF1 with CCR4-NOT results in gene activation, repression, or enhanced mRNA degradation did not reveal any specific sequence features. Therefore, the genomic context of the EBF1-binding sites may determine the functional outcome of the CCR4-NOT:EBF1 interaction. For example, the actions of the CCR4-NOT complex in mRNA deadenylation and decay have been found to involve RNA-binding proteins, such as PUF5 in yeast or tristetraprolin (TPP) in humans (Lee et al. 2010; Sandler et al. 2011). These proteins bind to the AU-rich elements in the $3^{\prime}$ untranslated region (UTR) and interact with components of the CCR4-NOT complex. Thus, the functional outcome of the EBF1:CCR4-NOT interaction may depend on additional regulatory inputs.

Analysis of the potential of EBF1H240A to induce B-cell differentiation in complementation assays of Ebf1-deficient progenitors indicated that early pro-B cells are generated at a frequency similar to EBF1wt transduced 
progenitors, whereas late pro-B/pre-B cells were less efficiently generated. This defect was not due to an altered cell proliferation or increased apoptosis and could be accounted for by the reduced expression of EBF1-regulated genes encoding components of the pre-BCR. In particular, the expression of the Ig surrogate light chain genes VpreB and Igl11 (encoding $\lambda 5$ ) is down-regulated in EBF1H240Aexpressing cells relative to EBF1wt-expressing cells. In addition, we observed a reduced expression of Rag1, Pou2af1 (encoding $\mathrm{OcaB}$ ), and $M z b 1$, encoding an ER-resident cochaperone that influences the folding of the Ig $\mu$ heavy chain and helps to buffer the effects of DNA damage in early B-lineage cells (Rosenbaum et al. 2014). In support of a role of the interaction of the CCR4-NOT complex with EBF1, we observed a similar developmental block in $C n o t 3^{f l / f 1} R E R T^{C r e}$ mice in which the second and third exons were deleted upon tamoxifen treatment. RNA-seq analysis of pro-B cells from tamoxifen-treated Cnot $3^{\text {fl/fl }}$ $R E R T^{C r e}$ mice indicated that Igll1 and VpreB were down-regulated relative to tamoxifen-treated $\mathrm{Cnot}^{+/+}$ RERT $T^{\text {Cre }}$ mice. Notably, in pro-B cells of Cnot $3^{f l / f l}$ $R E R T^{C r e}$ mice, we also observed a down-regulation of Ebf1 and Pax5 transcription and a reduction in their protein levels. These changes in EBF1 and Pax5 expression may be accounted for by an impaired autoregulation of Ebf1 and the impaired activation of Pax5 by EBF1 (Smith et al. 2002; Roessler et al. 2007; Decker et al. 2009). Thus, the impaired differentiation of Cnot3-deficient pro-B cells could be due to a combined effect on the expression of important transcriptional regulators and components of the pre-BCR.

During the course of our study, an analysis of Cnot3 deficiency in B cells using the $m b 1^{\text {Cre }}$ mouse line was reported (Inoue et al. 2015). This analysis revealed a defect in pro-B-cell differentiation similar to that observed in our study. In particular, Inoue et al. (2015) observed a preferential block in distal $\mathrm{V}(\mathrm{D}) \mathrm{J}$ recombination of the Igh locus and detected a destabilized expression of $p 53$. In our analysis of $C n o t 3^{f l / f 1} R E R T^{C r e}$ mice, we also observed a reduced distal but not proximal V(D)J recombination of the $I g H$ locus in pro-B cells. In contrast to the study by Inoue et al. (2015), however, we detected a markedly impaired expression of Pax5 protein, which has been shown to be required for distal $\mathrm{V}(\mathrm{D}) \mathrm{J}$ recombination (Fuxa et al. 2004). Therefore, we suggest that the effect of Cnot3 deficiency on Igh recombination is indirect via a reduced autoregulation of $E b f 1$ and reduced expression of Pax5. Moreover, the altered expression of p53 is not observed in Cnot $^{\text {fl/fl }}{ }^{R E R T} T^{\text {Cre }}$ mice and may be linked to the genotoxic stress observed in pro-B cells of the $\mathrm{mb1}^{\mathrm{Cre}}$ mouse line (Rosenbaum et al. 2014).

In conclusion, our study provides insight into the genespecific recruitment of the CCR4-NOT complex via interaction with EBF1. The interaction of EBF1 with the CNOT3 subunit of the CCR4-NOT complex allows for a diversified regulation of EBF1 target genes, including transcription and mRNA stability, and suggests that the gene-specific recruitment of the CCR4-NOT complex helps to coordinate transcriptional and post-transcriptional processes.

\section{Materials and methods}

Mice

All mouse experiments were carried out in accordance with the guidelines of the Federation of European Laboratory Animal Science Association (FELASA) following legal approval by the regional authorities. The generation of the $\operatorname{Cnot} 3^{f 1 / f 1}$ mice carrying loxP sites flanking the second and third exons of the Cnot3 locus will be described elsewhere (K Kuba and Y Imai, unpubl.). The Mzb1 transgene was generated by inserting the $M z b 1$ cDNA into a plasmid containing the intronic and $3^{\prime} \kappa$ enhancers and the $V \kappa 21$ promoter.

\section{Cell culture and retroviral transduction}

HEK293 cells were cultured in DMEM supplemented with $10 \%$ FCS and $1 \%$ PSG. 38B9 pro-B cells were cultured in RPMI-1640 medium supplemented with $10 \%$ FCS, $1 \%$ PSG, and $50 \mu \mathrm{M} \beta$ mercaptoethanol. $E b f 1^{-/-} \mathrm{c}-\mathrm{Kit}^{+}$progenitor cells were isolated from $E b f 1^{-/-}$fetal livers by staining with biotinylated c-Kit (BD Biosciences) and further addition of streptavidin beads (Miltenyi Biotec). The c-Kit ${ }^{+}$progenitors were enriched by an AutoMACS separator and subsequently plated on OP9 feeders in OptiMEM medium supplemented with 4\% FCS, $1 \%$ PSG, $50 \mu \mathrm{M} \beta$-mercaptoethanol, $10 \mathrm{ng} / \mathrm{mL} \mathrm{SCF}, 10 \mathrm{ng} / \mathrm{mL}$ Flt $3 \mathrm{~L}$, and $5 \mathrm{ng} / \mathrm{mL}$ IL-7. Retroviral transduction of pMys-IRES-GFP (vector), pMys-EBF1wtIRES-GFP, and pMys-EBF1H240A-IRES-GFP was performed as described previously (Treiber et al. 2010b). GFP-positive cells were sorted $36 \mathrm{~h}$ after transduction and further cultured on OP9 feeders. Reconstitution of $E b f 1^{f 1 / f 1} R E R T^{\text {Cre }}$ pro-B cells with EBF1wt or EBF1H240A was performed as described in Boller et al. (2016).

\section{Strep-Tactin pull-down}

Cells expressing the Strep-tagged protein were harvested, washed with PBS, and suspended in coimmunoprecipitation lysis buffer (20 mM Hepes at pH 7.6, 90 mM NaCl, 1 mM DTT, 5\% [v/v] glycerol, $0.1 \%$ [v/v] NP-40). One milligram of protein in $1 \mathrm{~mL}$ of lysate was used for each pull-down. Fifty microliters of washed StrepTactin beads was added to the protein solution. After incubation for $1 \mathrm{~h}$ at $4^{\circ} \mathrm{C}$, the flowthrough and Strep-Tactin beads were separated by a magnetic separation stand. The flowthrough was removed, and the beads were washed twice with $1 \mathrm{~mL}$ of coimmunoprecipitation buffer. The bound proteins were eluted by dissolving the beads in $20 \mu \mathrm{L}$ of Strep elution buffer (coimmunoprecipitation lysis buffer $+10 \mathrm{mM}$ biotin) in a thermo shaker at $1000 \mathrm{rpm}$ for $5 \mathrm{~min}$ at $25^{\circ} \mathrm{C}$. The eluent was used for the following experiments.

\section{Immunoblot analysis, immunoprecipitation, and ChIP}

Immunoblotting was performed with the following antibodies: anti-Flag (clone M2; Sigma), anti-HA (clone 3F10; Roche), antiGAPDH (clone 6C5; Calbiochem), anti-Pax5 (clone C-20; Santa Cruz Biotechnology), anti-CNOT3 (clone E1L9S; Cell Signaling), anti-CNOT2 (Cell Signaling), anti-CNOT7 (clone 18W; Santa Cruz Biotechnology), and anti-EBF1 (clone 7G4). For immunoprecipitation of EBF1 and EBF1-associated proteins, we used monoclonal (clone 7G4) and polyclonal (1C) anti-EBF1 antibodies. For visualization in the gel, $2 \%-5 \%$ of input and $10 \%-20 \%$ of eluate were used to detect the immunoprecipitation, and $80 \%-90 \%$ of eluate was used to determine the cobound proteins. ChIP was performed as described previously in detail (Boller et al. 2016). 


\section{Act $D$ treatment and analysis of $m R N A$ stability}

Act D was added directly to the culture medium to a concentration of $100 \mu \mathrm{M}$, and cells were harvested at different time points. The RNA was extracted from Trizol reagent, and cDNA was synthesized for the further gene expression analysis.

\section{Flow cytometric analysis}

For in vitro analysis, EBF1wt or EBF1H240A transduced cells were stained with various pro-B-cell markers for flow cytometric analysis. For in vivo analysis, single-cell suspensions from bone marrow or spleens were stained with the fluorochrome-conjugated antibodies purchased from BD Pharmingen or eBioscience and were analyzed using BD LSRII. The data were processed and evaluated by FlowJo software. Anti-CD19 (1D3), BP1 (BP-1), CD25 (PC61) HSA (M1/69), CD43 (R2/60), B220 (RA-6B2), CD93 (AA4.1), CD23 (B3B4), CD21 (7G6), and $\alpha$-IgM $\mu$ (Jackson ImmunoResearch, \#115-175-075) were used.

\section{Statistical analysis}

All data are shown as mean value \pm standard error of the mean and were tested statistically using a two-tailed Student's $t$-test or ANOVA. All figures and statistical analyses were generated using Microsoft Excel or Prism 5. $P<0.05$ was considered to indicate statistical significance.

SILAC-based proteomics of EBF1-associated proteins, microarray analysis, RNA-seq analysis, and ChIP-seq analysis are described in detail in the Supplemental Material.

The microarray, RNA-seq, and ChIP-seq data reported here were deposited at the National Center for Biotechnology Information under the accession number GSE87637.

\section{Acknowledgments}

We are grateful to Franziska Ludin and Ingrid Falk for technical help. We thank members of the Grosschedl laboratory for discussions, and Eirini Trompouki for critical reading of the manuscript. We thank Marika Rott for assistance in the manuscript preparation. Research at R.G.'s laboratory is supported by funds from the Max Planck Society and the German Research Foundation.

\section{References}

Adolfsson J, Mansson R, Buza-Vidas N, Hultquist A, Liuba K, Jensen CT, Bryder D, Yang L, Borge OJ, Thoren LA, et al. 2005. Identification of Flt3+ lympho-myeloid stem cells lacking erythro-megakaryocytic potential a revised road map for adult blood lineage commitment. Cell 121: 295-306.

Banerjee A, Northrup D, Boukarabila H, Jacobsen SE, Allman D. 2013. Transcriptional repression of Gata3 is essential for early B cell commitment. Immunity 38: 930-942.

Bhaskar V, Basquin J, Conti E. 2015. Architecture of the ubiquitylation module of the yeast Ccr4-Not complex. Structure 23: 921-928.

Boller S, Grosschedl R. 2014. The regulatory network of B-cell differentiation: a focused view of early B-cell factor 1 function. Immunol Rev 261: 102-115.

Boller S, Ramamoorthy S, Akbas D, Nechanitzky R, Burger L, Murr R, Schübeler D, Grosschedl R. 2016. Pioneering activity of the C-terminal domain of EBF1 shapes the chromatin landscape for B cell programming. Immunity 44: 527-541.
Bregman A, Avraham-Kelbert M, Barkai O, Duek L, Guterman A, Choder M. 2011. Promoter elements regulate cytoplasmic mRNA decay. Cell 147: 1473-1483.

Chen J, Chiang YC, Denis CL. 2002. CCR4, a 3'-5' poly(A) RNA and ssDNA exonuclease, is the catalytic component of the cytoplasmic deadenylase. EMBO I 21: 1414-1426.

Chowdhury D, Sen R. 2004. Mechanisms for feedback inhibition of the immunoglobulin heavy chain locus. Curr Opin Immunol 16: 235-240.

Collart MA, Struhl K. 1994. NOT1(CDC39), NOT2(CDC36), NOT3, and NOT4 encode a global-negative regulator of transcription that differentially affects TATA-element utilization. Genes Dev 8: 525-537.

Collart MA, Panasenko OO, Nikolaev SI. 2013. The Not3/5 subunit of the Ccr4-Not complex: a central regulator of gene expression that integrates signals between the cytoplasm and the nucleus in eukaryotic cells. Cell Signal 25: 743-751.

Decker T, Pasca di Magliano M, McManus S, Sun Q, Bonifer C, Tagoh H, Busslinger M. 2009. Stepwise activation of enhancer and promoter regions of the B cell commitment gene Pax5 in early lymphopoiesis. Immunity 30: 508-520.

Dutta A, Babbarwal V, Fu J, Brunke-Reese D, Libert DM, Willis J, Reese JC. 2015. Ccr4-Not and TFIIS function cooperatively to rescue arrested RNA polymerase II. Mol Cell Biol 35: 1915-1925.

Fuxa M, Skok J, Souabni A, Salvagiotto G, Roldan E, Busslinger M. 2004. Pax5 induces V-to-DJ rearrangements and locus contraction of the immunoglobulin heavy-chain gene. Genes Dev 18: 411-422.

Gao H, Lukin K, Ramirez J, Fields S, Lopez D, Hagman J. 2009. Opposing effects of SWI/SNF and Mi-2/NuRD chromatin remodeling complexes on epigenetic reprogramming by EBF and Pax5. Proc Nat1 Acad Sci 106: 11258-11263.

Goler-Baron V, Selitrennik M, Barkai O, Haimovich G, Lotan R, Choder M. 2008. Transcription in the nucleus and mRNA decay in the cytoplasm are coupled processes. Genes Dev 22: 2022-2027.

Hagman J, Ramírez J, Lukin K. 2012. B lymphocyte lineage specification, commitment and epigenetic control of transcription by early B cell factor 1 . In Epigenetic regulation of lymphocyte development (ed. Murre C), pp. 17-38. Springer, Berlin.

Hata A, Seoane J, Lagna G, Montalvo E, Hemmati-Brivanlou A, Massagué J. 2000. OAZ uses distinct DNA- and protein-binding zinc fingers in separate BMP-Smad and Olf signaling pathways. Cell 100: 229-240.

Hentges KE, Weiser KC, Schountz T, Woodward LS, Morse HC III, Justice MJ. 2004. Evi3, a zinc-finger protein related to EBFAZ, regulates EBF activity in B-cell leukemia. Oncogene 24: $1220-1230$.

Hobeika E, Thiemann S, Storch B, Jumaa H, Nielsen PJ, Pelanda R, Reth M. 2006. Testing gene function early in the B cell lineage in mb1-cre mice. Proc Natl Acad Sci 103: 13789-13794.

Hu G, Kim J, Xu Q, Leng Y, Orkin SH, Elledge SJ. 2009. A genomewide RNAi screen identifies a new transcriptional module required for self-renewal. Genes Dev 23: 837-848.

Inlay MA, Bhattacharya D, Sahoo D, Serwold T, Seita J, Karsunky H, Plevritis SK, Dill DL, Weissman IL. 2009. Ly6d marks the earliest stage of B-cell specification and identifies the branchpoint between B-cell and T-cell development. Genes Dev 23: 2376-2381.

Inoue $\mathrm{T}$, Morita M, Hijikata A, Fukuda-Yuzawa $\mathrm{Y}$, Adachi S, Isono K, Ikawa T, Kawamoto $\mathrm{H}$, Koseki $\mathrm{H}$, Natsume $\mathrm{T}$, et al. 2015. CNOT3 contributes to early B cell development by controlling Igh rearrangement and p53 mRNA stability. J Exp Med 212: 1465-1479. 
Kiviranta R, Yamana K, Saito H, Ho DK, Laine J, Tarkkonen K, Nieminen-Pihala V, Hesse E, Correa D, Määttä J, et al. 2013. Coordinated transcriptional regulation of bone homeostasis by Ebf 1 and Zfp521 in both mesenchymal and hematopoietic lineages. J Exp Med 210: 969-985.

Kondo M, Weissman IL, Akashi K. 1997. Identification of clonogenic common lymphoid progenitors in mouse bone marrow. Cell 91: 661-672.

Kruk JA, Dutta A, Fu J, Gilmour DS, Reese JC. 2011. The multifunctional Ccr4-Not complex directly promotes transcription elongation. Genes Dev 25: 581-593.

Lau NC, Kolkman A, van Schaik FMA, Mulder KW, Pijnappel WWWP, Heck AJR, Timmers HTM. 2009. Human Ccr4Not complexes contain variable deadenylase subunits. Biochem J 422: 443-453.

Lee D, Ohn T, Chiang Y-C, Quigley G, Yao G, Liu Y, Denis CL. 2010. PUF3 acceleration of deadenylation in vivo can operate independently of CCR4 activity, possibly involving effects on the PAB1-mRNP Structure. I Mol Biol 399: 562-575.

Lemaire M, Collart MA. 2000. The TATA-binding protein-associated factor yTaf(II) $19 \mathrm{p}$ functionally interacts with components of the global transcriptional regulator Ccr4-Not complex and physically interacts with the Not5 subunit. I Biol Chem 275: 26925-26934.

Lin H, Grosschedl R. 1995. Failure of B-cell differentiation in mice lacking the transcription factor EBF. Nature 376: 263-267.

Lin YC, Jhunjhunwala S, Benner C, Heinz S, Welinder E, Mansson R, Sigvardsson M, Hagman J, Espinoza CA, Dutkowski J, et al. 2010. A global network of transcription factors, involving E2A, EBF1 and Foxo1, that orchestrates B cell fate. Nat Immunol 11: 635-643.

Liu HY, Badarinarayana V, Audino DC, Rappsilber J, Mann M, Denis CL. 1998. The NOT proteins are part of the CCR4 transcriptional complex and affect gene expression both positively and negatively. EMBO J 17: 1096-1106.

Lotan R, Bar-On VG, Harel-Sharvit L, Duek L, Melamed D, Choder M. 2005. The RNA polymerase II subunit Rpb4p mediates decay of a specific class of mRNAs. Genes Dev 19: 3004-3016.

Lukin K, Fields S, Guerrettaz L, Straign D, Rodriguez V, Zandi S, Månsson R, Cambier JC, Sigvardsson M, Hagman J. 2011. A dose-dependent role for EBF1 in repressing non-B-cell-specific genes. Eur I Immunol 41: 1787-1793.

Maier H, Ostraat R, Gao H, Fields S, Shinton SA, Medina KL, Ikawa T, Murre C, Singh H, Hardy RR, et al. 2004. Early B cell factor cooperates with Runxl and mediates epigenetic changes associated with mb-1 transcription. Nat Immunol 5: 1069-1077.

Malik S, Roeder RG. 2010. The metazoan Mediator co-activator complex as an integrative hub for transcriptional regulation. Nat Rev Genet 11: 761-772.

Mansson R, Welinder E, Ahsberg J, Lin YC, Benner C, Glass CK, Lucas JS, Sigvardsson M, Murre C. 2012. Positive intergenic feedback circuitry, involving EBF1 and FOXO1, orchestrates B-cell fate. Proc Natl Acad Sci 109: 21028-21033.

Meier K, Brehm A. 2014. Chromatin regulation: how complex does it get? Epigenetics 9: 1485-1495.

Mercer Elinore M, Lin Yin C, Benner C, Jhunjhunwala S, Dutkowski J, Flores M, Sigvardsson M, Ideker T, Glass Christopher K, Murre C. 2011. Multilineage priming of enhancer repertoires precedes commitment to the $\mathrm{B}$ and myeloid cell lineages in hematopoietic progenitors. Immunity 35: 413-425.
Miller JE, Reese JC. 2012. Ccr4-Not complex: the control freak of eukaryotic cells. Crit Rev Biochem Mol Biol 47: 315-333.

Morita M, Oike Y, Nagashima T, Kadomatsu T, Tabata M, Suzuki T, Nakamura T, Yoshida N, Okada M, Yamamoto T. 2011. Obesity resistance and increased hepatic expression of catabolism-related mRNAs in $\operatorname{Cnot} 3^{+/-}$mice. EMBO I 30: 4678-4691.

Nakamura T, Yao R, Ogawa T, Suzuki T, Ito C, Tsunekawa N, Inoue K, Ajima R, Miyasaka T, Yoshida Y, et al. 2004. Oligoastheno-teratozoospermia in mice lacking Cnot7, a regulator of retinoid X receptor $\beta$. Nat Genet 36: 528-533.

Nechanitzky R, Akbas D, Scherer S, Gyory I, Hoyler T, Ramamoorthy S, Diefenbach A, Grosschedl R. 2013. Transcription factor EBF1 is essential for the maintenance of B cell identity and prevention of alternative fates in committed cells. Nat Immunol 14: 867-875.

Neely GG, Kuba K, Cammarato A, Isobe K, Amann S, Zhang LY, Murata M, Elmen L, Gupta V, Arora S, et al. 2010. A global in vivo Drosophila RNAi screen identifies NOT3 as a conserved regulator of heart function. Cell 141: 142-153.

Nutt SL, Kee BL. 2007. The transcriptional regulation of B cell lineage commitment. Immunity 26: 715-725.

Nutt SL, Heavey B, Rolink AG, Busslinger M. 1999. Commitment to the B-lymphoid lineage depends on the transcription factor Pax5. Nature 401: 556-562.

Panasenko OO. 2014. The role of the E3 ligase Not4 in cotranslational quality control. Front Genet 5: 141.

Pongubala JM, Northrup DL, Lancki DW, Medina KL, Treiber T, Bertolino E, Thomas M, Grosschedl R, Allman D, Singh H. 2008. Transcription factor EBF restricts alternative lineage options and promotes B cell fate commitment independently of Pax5. Nat Immunol 9: 203-215.

Ramírez J, Lukin K, Hagman J. 2010. From hematopoietic progenitors to B cells: mechanisms of lineage restriction and commitment. Curr Opin Immunol 22: 177-184.

Revilla IDR, Bilic I, Vilagos B, Tagoh H, Ebert A, Tamir IM, Smeenk L, Trupke J, Sommer A, Jaritz M, et al. 2012. The Bcell identity factor Pax5 regulates distinct transcriptional programmes in early and late B lymphopoiesis. EMBO I 31: 3130-3146.

Reynaud D, Demarco IA, Reddy KL, Schjerven H, Bertolino E, Chen Z, Smale ST, Winandy S, Singh H. 2008. Regulation of B cell fate commitment and immunoglobulin heavy-chain gene rearrangements by Ikaros. Nat Immunol 9: 927-936.

Roessler S, Gyory I, Imhof S, Spivakov M, Williams RR, Busslinger M, Fisher AG, Grosschedl R. 2007. Distinct promoters mediate the regulation of Ebf1 gene expression by interleukin7 and Pax5. Mol Cell Biol 27: 579-594.

Rosenbaum M, Andreani V, Kapoor T, Herp S, Flach H, Duchniewicz M, Grosschedl R. 2014. MZB1 is a GRP94 cochaperone that enables proper immunoglobulin heavy chain biosynthesis upon ER stress. Genes Dev 28: 1165-1178.

Sanders SL, Jennings J, Canutescu A, Link AJ, Weil PA. 2002. Proteomics of the eukaryotic transcription machinery: identification of proteins associated with components of yeast TFIID by multidimensional mass spectrometry. Mol Cell Biol 22: 4723-4738.

Sandler H, Kreth J, Timmers HTM, Stoecklin G. 2011. Not1 mediates recruitment of the deadenylase Caf1 to mRNAs targeted for degradation by tristetraprolin. Nucleic Acids Res 39: 4373-4386.

Schneider R, Grosschedl R. 2007. Dynamics and interplay of nuclear architecture, genome organization, and gene expression. Genes Dev 21: 3027-3043. 
Schwickert TA, Tagoh H, Gultekin S, Dakic A, Axelsson E, Minnich M, Ebert A, Werner B, Roth M, Cimmino L, et al. 2014. Stage-specific control of early B cell development by the transcription factor Ikaros. Nat Immunol 15: 283-293.

Singh H, Khan AA, Dinner AR. 2014. Gene regulatory networks in the immune system. Trends Immunol 35: 211-218.

Siponen MI, Wisniewska M, Lehtio L, Johansson I, Svensson L, Raszewski G, Nilsson L, Sigvardsson M, Berglund H. 2010. Structural determination of functional domains in early Bcell factor (EBF) family of transcription factors reveals similarities to Rel DNA-binding proteins and a novel dimerization motif. J Biol Chem 285: 25875-25879.

Smith EM, Gisler R, Sigvardsson M. 2002. Cloning and characterization of a promoter flanking the early $\mathrm{B}$ cell factor (EBF) gene indicates roles for E-proteins and autoregulation in the control of EBF expression. J Immunol 169: 261-270.

Thal MA, Carvalho TL, He T, Kim H-G, Gao H, Hagman J, Klug CA. 2009. Ebf1-mediated down-regulation of Id2 and Id 3 is essential for specification of the B cell lineage. Proc Natl Acad Sci 106: 552-557.

Treiber N, Treiber T, Zocher G, Grosschedl R. 2010a. Structure of an Ebf1:DNA complex reveals unusual DNA recognition and structural homology with Rel proteins. Genes Dev 24: 2270-2275.

Treiber T, Mandel EM, Pott S, Gyory I, Firner S, Liu ET, Grosschedl R. 2010b. Early B cell factor 1 regulates B cell gene networks by activation, repression, and transcription- independent poising of chromatin. Immunity 32: 714-725.
Tucker M, Valencia-Sanchez MA, Staples RR, Chen J, Denis CL, Parker R. 2001. The transcription factor associated Ccr4 and Caf1 proteins are components of the major cytoplasmic mRNA deadenylase in Saccharomyces cerevisiae. Cell 104: 377-386.

Villanyi Z, Ribaud V, Kassem S, Panasenko OO, Pahi Z, Gupta I, Steinmetz L, Boros I, Collart MA. 2014. The Not5 subunit of the Ccr4-Not complex connects transcription and translation. PLoS Genet 10: e1004569.

Winkler GS, Mulder KW, Bardwell VJ, Kalkhoven E, Timmers HTM. 2006. Human Ccr4-Not complex is a ligand-dependent repressor of nuclear receptor-mediated transcription. EMBO J 25: 3089-3099.

Zandi S, Mansson R, Tsapogas P, Zetterblad J, Bryder D, Sigvardsson M. 2008. EBF1 is essential for B-lineage priming and establishment of a transcription factor network in common lymphoid progenitors. I Immunol 181: 3364 3372 .

Zandi S, Åhsberg J, Tsapogas P, Stjernberg J, Qian H, Sigvardsson M. 2012. Single-cell analysis of early B-lymphocyte development suggests independent regulation of lineage specification and commitment in vivo. Proc Natl Acad Sci 109: 15871-15876.

Zwartjes CGM, Jayne S, Van Den Berg DLC, Timmers HTM. 2004. Repression of promoter activity by CNOT2, a subunit of the transcription regulatory Ccr4-Not complex. I Biol Chem 279: 10848-10854. 


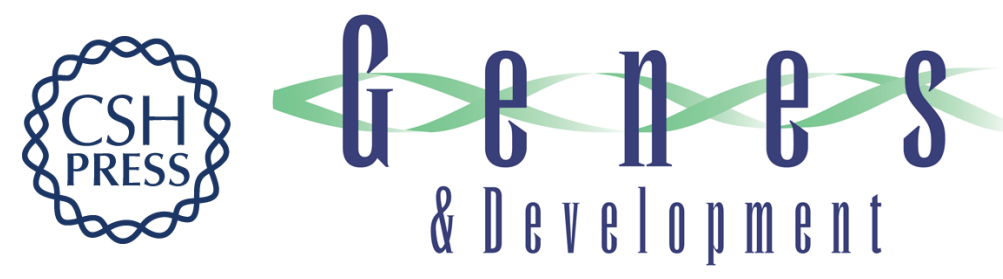

\section{Interaction of CCR4-NOT with EBF1 regulates gene-specific transcription and mRNA stability in B lymphopoiesis}

Cheng-Yuan Yang, Senthilkumar Ramamoorthy, Sören Boller, et al.

Genes Dev. 2016, 30: originally published online November 2, 2016

Access the most recent version at doi:10.1101/gad.285452.116

\section{Supplemental http://genesdev.cshlp.org/content/suppl/2016/11/02/gad.285452.116.DC1 \\ Material}

References This article cites 67 articles, 31 of which can be accessed free at:

http://genesdev.cshlp.org/content/30/20/2310.full.html\#ref-list-1

Creative This article, published in Genes \& Development, is available under a Creative Commons

Commons License (Attribution-NonCommercial 4.0 International), as described at

License http://creativecommons.org/licenses/by-nc/4.0/.

Email Alerting Receive free email alerts when new articles cite this article - sign up in the box at the top Service right corner of the article or click here.

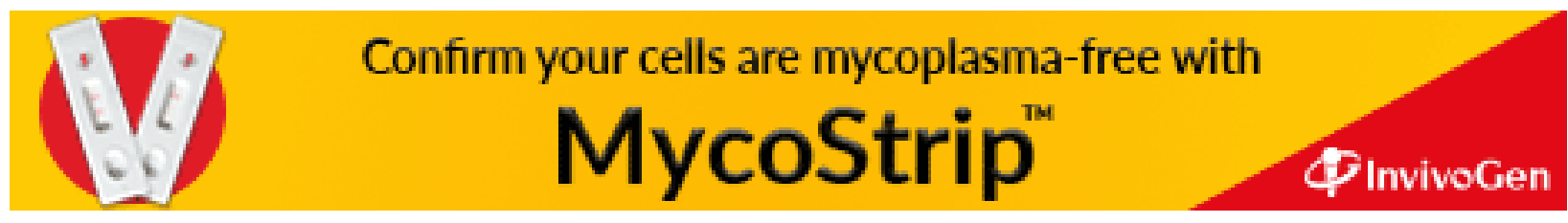

